\title{
SIMPLIFYING BRANCHED COVERING SURFACE-KNOTS BY AN ADDITION OF 1-HANDLES WITH CHART LOOPS
}

\author{
INASA NAKAMURA
}

\begin{abstract}
A branched covering surface-knot over an oriented surfaceknot $F$ is a surface-knot in the form of a branched covering over $F$. A branched covering surface-knot over $F$ is presented by a graph called a chart on a surface diagram of $F$. For a branched covering surface-knot, an addition of 1-handles equipped with chart loops is a simplifying operation which deforms the chart to the form of the union of free edges and 1-handles with chart loops. We investigate properties of such simplifications.
\end{abstract}

\section{INTRODUCTION}

A surface-knot is the image of a smooth embedding of a closed surface into the Euclidean 4-space $\mathbb{R}^{4}[2,5]$. In this paper, we consider oriented surface-knots. For a surface-knot $F$, we consider a surface in the form of a covering over $F$, called a branched covering surface-knot or a 2-dimensional braid over $F[7,8]$. The first aim of this paper is to announce that we change the name of a "2-dimensional braid" to a "branched covering surface-knot" (see Section 2). Two branched covering surface-knots over $F$ are equivalent if one is carried to the other by an ambient isotopy of $\mathbb{R}^{4}$ whose restriction to a tubular neighborhood of $F$ is fiber-preserving. A branched covering surface-knot over $F$, denoted by $(F, \Gamma)$, is presented by a graph $\Gamma$ called a chart on a surface diagram of $F[8]$.

In [9], we showed that a branched covering surface-knot deforms to a simplified form in terms of charts by an addition of 1-handles with chart loops. The second aim of this paper is to investigate further such simplifications of branched covering surface-knots.

Let $B^{2}$ be a unit 2-disk and let $I=[0,1]$. A 1-handle is a 3-ball $h=B^{2} \times I$ smoothly embedded in $\mathbb{R}^{4}$ such that $h \cap F=B^{2} \times \partial I$. In particular, a 1handle $h$ is said to be trivial if it is embedded in a 3 -ball $B^{3}$ such that $F \cap B^{3}$ is a 2-disk. The surface-knot obtained from $F$ by a 1-handle addition along $h$ is the surface

$$
\left(F-\left(\operatorname{Int} B^{2} \times \partial I\right)\right) \cup\left(\partial B^{2} \times I\right),
$$

which is denoted by $F+h$. In this paper, we assume that $h$ is orientable, that is, $F+h$ is orientable, and we give $F+h$ the orientation induced from that of $F$. Further, we assume that 1-handles are trivial. Let $h=B^{2} \times I$ be a 1-handle. We call $B^{2} \times\{0\}$ and $B^{2} \times\{1\}$ the ends of $h$. When both ends of $h$ are on a 2-disk $E$ in $F$, we determine the core loop of $h$ as follows. Let $\rho(t), t \in I$ be an oriented path in $\partial B^{2} \times I \subset h$ with the orientation of $I$ such

2010 Mathematics Subject Classification. Primary 57Q45; Secondary 57Q35.

Key words and phrases. surface-knot; 2-dimensional braid; chart; 1-handle. 
that $\rho(t) \in \partial B^{2} \times\{t\}, t \in I$. We define the core loop of $h$ by the oriented closed path in $F+h$ obtained from $\rho(t), t \in[0,1]$ by connecting the initial and terminal points $\rho(0)$ and $\rho(1)$ by a simple arc in $E$, with the induced orientation. We determine the cocore of a 1-handle $h$ by the oriented closed path $\partial B^{2} \times\{0\} \subset h$, with the orientation of $\partial B^{2}$. Further, we determine the base point of the core loop and the cocore of $h$ by their intersection point. See Fig. 1. For a set of 1-handles, we add a condition that core loops are mutually disjoint.
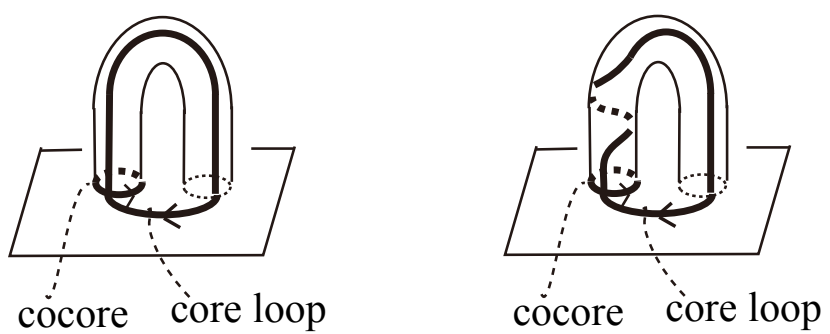

Figure 1. The core loop and the cocore of a 1-handle. There are two types.

Assumption 1. In this paper, for simplicity, we assume that all 1-handles are trivial, and we assume that a surface-knot $F$ is an unknotted surfaceknot with one component in the standard form, that is, $F$ is in the form of the boundary of a handlebody in $\mathbb{R}^{3} \times\{0\} \subset \mathbb{R}^{4}$.

A 1-handle admits two types framings, presented by the core loop and the cocore as indicated in Fig. 1 1, 6, see also [9, Lemma 4.2]. In this paper, for simplicity, we do not distinguish the framings. We consider a 1-handle $h$ attached to a 2 -disk $E$. For commutative braids $a$ and $b$, we denote by $h(a, b)$ a covering surface over $h$ whose cocore with the given orientation and core loop with the reversed orientation present the braids $a$ and $b$, respectively. Such a chart is drawn on the union of $h$ and neighborhoods of the core loop and the cocore in $E$, and this chart is unique up to C-move equivalence [7], where we assume that $E$ has no edges nor vertices of a chart except those coming from $h(a, b)$. Hence, the notation $h(a, b)$ is well-defined, under the condition that we assign the place where we attach the 1-handle. We call the chart on $E+h$ a chart of a 1-handle, and we call $h(a, b)$ a 1-handle with a chart, or simply a 1-handle. A chart loop is a closed path consisting of an edge of a chart or diagonal edges of a chart connected with vertices of degree 4 (crossings). In particular, when a chart of a 1-handle consists of chart loops, we call such a 1-handle a 1-handle with chart loops.

Remark 1.1. We repeat that we do not distinguish the framings of 1handles. If we take the core loop and the cocore of a 1-handle as indicated in the left figure of Fig. 1, then a 1-handle $h(a, b)$ with the core loop and the cocore as in the right figure of Fig. 1 is denoted by $h(a, a b)$. For simplicity, we denote both types of 1-handles by $h(a, b)$.

An edge of a chart is called a free edge if its end points are vertices of degree one (black vertices). Let $\Gamma_{0}$ be a chart consisting of a disjoint union 
of several free edges. For a branched covering surface-knot $\left(F, \Gamma_{0}\right)$ and 1handles with charts $h\left(a_{1}, b_{1}\right), \ldots, h\left(a_{g}, b_{g}\right)$ attached to a 2 -disk $E$ in $F$, where there are no edges nor vertices of charts on $E$ except those of the attached 1-handles, we denote the branched covering surface-knot which is the result of the 1-handle addition by $\left(F^{\prime}, \Gamma^{\prime}\right)=\left(F, \Gamma_{0}\right)+\sum_{i=1}^{g} h\left(a_{i}, b_{i}\right)$. Note that since $\Gamma_{0}$ is a disjoint union of free edges, the presentation is well-defined.

We showed in [9] the following results. Under Assumption 1, the results are written as follow. Let $N$ be a positive integer.

Theorem 1.2 ([9, Theorem 1.6]). Let $(F, \Gamma)$ be a branched covering surfaceknot of degree $N$. By an addition of finitely many 1-handles in the form $h\left(\sigma_{i}, e\right)$ or $h(e, e)(i \in\{1, \ldots, N-1\})$, to appropriate places in $F,(F, \Gamma)$ deforms to

$$
\left(F, \Gamma_{0}\right)+\sum_{k} h\left(\sigma_{i_{k}}, e\right)+\sum_{l} h\left(\sigma_{i_{l}}, \sigma_{j_{l}}^{\epsilon_{l}}\right)+\sum h(e, e),
$$

where $i_{k}, i_{l}, j_{l} \in\{1, \ldots, N-1\},\left|i_{l}-j_{l}\right|>1$ and $\epsilon_{l} \in\{+1,-1\}$, and $\Gamma_{0}$ is a chart consisting of several (maybe no) free edges.

In particular, by an addition of 1-handles $\sum_{i=1}^{N-1} h\left(\sigma_{i}, e\right)$ and finitely many $h(e, e)$, to a fixed 2-disk in $F,(F, \Gamma)$ deforms to

$$
\left(F, \Gamma_{0}\right)+\sum_{i=1}^{N-1} h\left(\sigma_{i}, e\right)+\sum_{l} h\left(\sigma_{i_{l}}, \sigma_{j_{l}}^{\epsilon_{l}}\right)+\sum h(e, e),
$$

where $i_{l}, j_{l} \in\{1, \ldots, N-1\},\left|i_{l}-j_{l}\right|>1$ and $\epsilon_{l} \in\{+1,-1\}$, and $\Gamma_{0}$ is a chart consisting of several free edges.

Definition 1.3. We call $(F, \Gamma)$ in the form $(1.1)$ a branched covering surfaceknot in a weak simplified form, and we call the minimal number of 1-handles necessary to deform $(F, \Gamma)$ to the form (1.1) the weak simplifying number of $(F, \Gamma)$, which is denoted by $u_{w}(F, \Gamma)$.

Theorem 1.4 ([9, Theorem 1.8]). Let $(F, \Gamma)$ be a branched covering surfaceknot of degree $N$. By an addition of finitely many 1-handles in the form $h\left(\sigma_{i}, e\right), h\left(\sigma_{i}, \sigma_{j}^{\epsilon}\right)$ or $h(e, e)(i, j \in\{1, \ldots, N-1\},|i-j|>1, \epsilon \in\{+1,-1\})$, to appropriate places in $F,(F, \Gamma)$ deforms to

$$
\left(F, \Gamma_{0}\right)+\sum_{k} h\left(\sigma_{i_{k}}, e\right)+\sum h(e, e),
$$

where $i_{k} \in\{1, \ldots, N-1\}$ and $\Gamma_{0}$ is a chart consisting of several free edges.

In particular, by an addition of 1-handles $\sum_{i=1}^{N-1} h\left(\sigma_{i}, e\right)$ and finitely many 1 -handles in the form $h\left(\sigma_{i}, \sigma_{j}^{\epsilon}\right)(i, j \in\{1, \ldots, N-1\},|i-j|>1, \epsilon \in$ $\{+1,-1\})$ or $h(e, e)$, to a fixed 2-disk in $F,(F, \Gamma)$ deforms to

$$
\left(F, \Gamma_{0}\right)+\sum_{i=1}^{N-1} h\left(\sigma_{i}, e\right)+\sum h(e, e),
$$

where $\Gamma_{0}$ is a chart consisting of several free edges.

Definition 1.5. We call $(F, \Gamma)$ in the form $(1.3)$ a branched covering surfaceknot in a simplified form, and we call the minimal number of 1-handles necessary to deform $(F, \Gamma)$ to the form $(1.3)$ the simplifying number of $(F, \Gamma)$, which is denoted by $u(F, \Gamma)$. 
Remark 1.6. Here, we give new names for $u_{w}(F, \Gamma)$ and $u(F, \Gamma)$. In [9], we called $u_{w}(F, \Gamma)$ and $u(F, \Gamma)$ the weak unbraiding number and the unbraiding number, respectively.

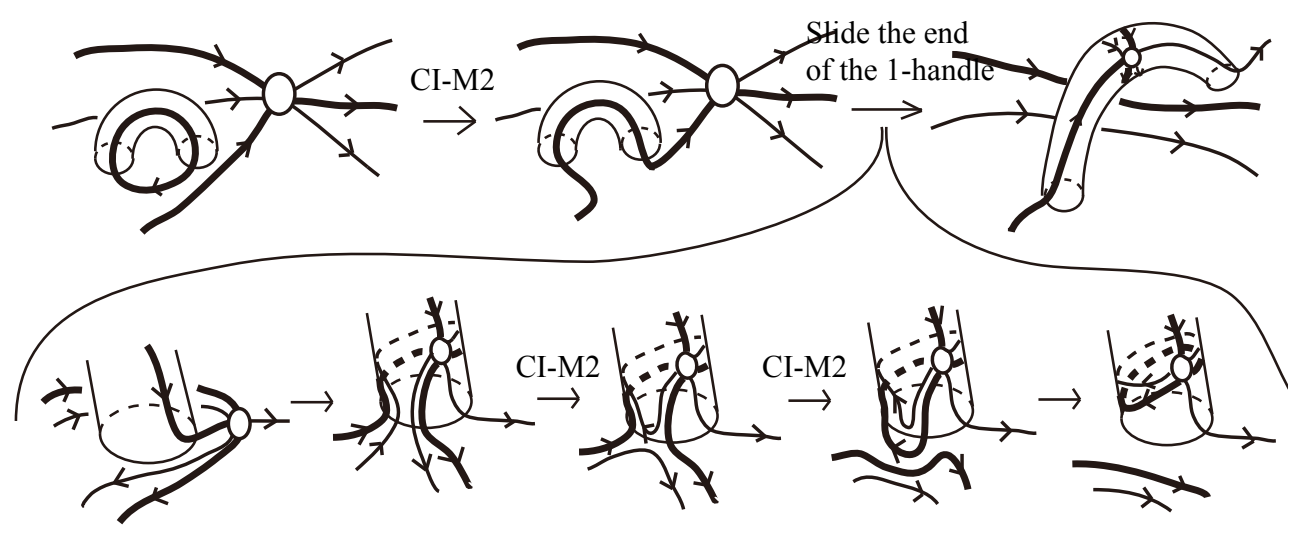

Figure 2. Collecting a white vertex on a 1-handle. For simplicity, we omit labels of chart edges.

Our results are as follow. Let $(F, \Gamma)$ be a branched covering surface-knot. Let $N$ be the degree of the chart $\Gamma$. We denote by $b(\Gamma), c(\Gamma)$, and $w(\Gamma)$ the numbers of black vertices, crossings, and white vertices, respectively.

In [9, Proposition 1.11], we obtained the inequality $u_{w}(F, \Gamma) \leq w(\Gamma)+$ $2 c(\Gamma)+N-1$. We showed it by deforming $(F, \Gamma)$ to a weak simplified form by sliding an end of a 1-handle to collect white vertices as indicated in Fig. 2 , and eliminating white vertices and then chart loops by a certain method; see [9]. By deforming $(F, \Gamma)$ to a weak simplified form by a new method, we obtain the following inequality.

Theorem 1.7. Let $(F, \Gamma)$ be a branched covering surface-knot of degree $N$. Let $b(\Gamma), c(\Gamma)$, and $w(\Gamma)$ be the numbers of black vertices, crossings, and white vertices of $\Gamma$, respectively. We have

$$
u_{w}(F, \Gamma) \leq \max \left\{\left\lfloor\frac{w(\Gamma)}{2}+\frac{b(\Gamma)}{4}(N-2)\right\rfloor, c(\Gamma)\right\}+N-1,
$$

where $\lfloor x\rfloor$ is the largest integer less than or equal to $x$. In particular, if $b(\Gamma)=0$, then

$$
u_{w}(F, \Gamma) \leq \max \left\{\frac{w(\Gamma)}{2}, c(\Gamma)\right\}+N-1 .
$$

Theorem 1.8. Let $(F, \Gamma)$ be a branched covering surface-knot of degree $N$. By an addition of finitely many 1-handles in the form $h\left(\sigma_{i}, e\right)$, or $h(e, e)$ $(i \in\{1, \ldots, N-1\})$, to appropriate places in $F,(F, \Gamma)$ deforms to

$$
\left(F, \Gamma_{0}\right)+\sum_{i=1}^{N-1} h\left(\sigma_{i}, e\right)+\sum h\left(\sigma_{1}, \sigma_{3}^{\epsilon}\right)+\sum h(e, e),
$$

where $\Gamma_{0}$ is a chart consisting of several free edges, and $\epsilon$ is a fixed sign valued in $\{+1,-1\}$. 
Theorem 1.9. Let $(F, \Gamma)$ be a branched covering surface-knot of degree $N$. $B y$ an addition of finitely many 1-handles in the form $h\left(\sigma_{i}, e\right)$, or $h(e, e)$ $(i \in\{1, \ldots, N-1\})$, to appropriate places in $F,(F, \Gamma)$ deforms to one of the followings:

$$
\left(F, \Gamma_{0}\right)+\sum_{i=1}^{N-1} h\left(\sigma_{i}, e\right)+\sum h(e, e)
$$

or

$$
\left(F, \Gamma_{0}\right)+h\left(\sigma_{1}, \sigma_{3}\right)+\sum_{i=2}^{N-1} h\left(\sigma_{i}, e\right)+\sum h(e, e),
$$

where $\Gamma_{0}$ is a chart consisting of several free edges.

In particular, if $w(\Gamma)=0$ or $b(\Gamma)=0$, then $(F, \Gamma)$ deforms to (1.8) if $c(\Gamma)$ is even, and (1.9) if $c(\Gamma)$ is odd. Here $w(\Gamma), b(\Gamma)$ and $c(\Gamma)$ are the numbers of white vertices, black vertices and crossings of $\Gamma$, respectively. We write down the case when $b(\Gamma)=0$ as follows. If $b(\Gamma)=0$, then $(F, \Gamma)$ deforms to

$$
(F, \emptyset)+\sum_{i=1}^{N-1} h\left(\sigma_{i}, e\right)+\sum h(e, e) \text {, if } c(\Gamma) \text { is even, }
$$

and

$$
(F, \emptyset)+h\left(\sigma_{1}, \sigma_{3}\right)+\sum_{i=2}^{N-1} h\left(\sigma_{i}, e\right)+\sum h(e, e), \text { if } c(\Gamma) \text { is odd }
$$

Corollary 1.10. Let $(F, \Gamma)$ be a branched covering surface-knot of degree $N$. Let $w(\Gamma)$ and $b(\Gamma)$ be the numbers of white vertices and black vertices of $\Gamma$, respectively. If $w(\Gamma)=0$, then

$$
u_{w}(F, \Gamma) \leq N
$$

Further,

$$
u_{w}(F, \Gamma) \leq \max \left\{1,\left\lfloor\frac{w(\Gamma)}{2}+\frac{b(\Gamma)}{4}(N-2)\right\rfloor\right\}+N-1,
$$

where $\lfloor x\rfloor$ is the largest integer less than or equal to $x$. In particular, if $b(\Gamma)=0$, then

$$
u_{w}(F, \Gamma) \leq \max \left\{1, \frac{w(\Gamma)}{2}\right\}+N-1 .
$$

Further, the inequalities 1.12, 1.13) and 1.14 also hold true for $u(F, \Gamma)$.

Corollary 1.11. Let $(F, \Gamma)$ be a branched covering surface-knot of degree $N$. Then

$$
u(F, \Gamma) \leq \max \left\{1, u_{w}(F, \Gamma)\right\}+N-1 .
$$

In [9, Proposition 1.10], we announced that $u_{w}(F, \Gamma) \leq u(F, \Gamma) \leq u_{w}(F, \Gamma)+$ $c_{\text {alg }}(\Gamma)$, where $c_{\text {alg }}(\Gamma)$ is the sum of the absolute values of the numbers each of which is the number of crossings of type $c_{i, j}$ minus that of type $c_{j, i}(i<j$, $i, j \in\{1, \ldots, N-1\})$; see Remark $\frac{5.6}{5}$. In the proof for $u_{w}(F, \Gamma) \leq u(F, \Gamma)$, 
we said it is obvious, but it is not. We thought that by an addition of 1handles in the form $h\left(\sigma_{i}, e\right)$ or $h(e, e)$, we first deform $(F, \Gamma)$ to a branched covering surface-knot $\left(F^{\prime}, \Gamma^{\prime}\right)$ in a weak simplified form (1.1), and then by an addition of 1-handles in the form $h\left(\sigma_{i}, \sigma_{j}^{\epsilon}\right)(|i-j|>1, \epsilon \in\{+1,-1\})$, we deform $\left(F^{\prime}, \Gamma^{\prime}\right)$ to a simplified form $(1.3)$. We forgot that we can add $h\left(\sigma_{i}, \sigma_{j}^{\epsilon}\right)$ from the first. We correct the statement to a conjecture.

Conjecture 1.12. Let $(F, \Gamma)$ be a branched covering surface-knot. We conjecture that

$$
u_{w}(F, \Gamma) \leq u(F, \Gamma) .
$$

For $u(F, \Gamma) \leq u_{w}(F, \Gamma)+c_{\text {alg }}(\Gamma)$, the proof given in [9] was for a special case; see Remark 5.6. We correct the statement to a conjecture.

Conjecture 1.13. Let $(F, \Gamma)$ be a branched covering surface-knot of degree $N$. We conjecture that

$$
u(F, \Gamma) \leq u_{w}(F, \Gamma)+c_{\text {alg }}(\Gamma),
$$

where $c_{\mathrm{alg}}(\Gamma)$ is the sum of the absolute values of the numbers each of which is the number of crossings of type $c_{i, j}$ minus that of type $c_{j, i}(i<j, i, j \in$ $\{1, \ldots, N-1\})$; see Remark 5.6. Further, by the proof of Corollary 1.11. we conjecture that

$$
u(F, \Gamma) \leq \max \left\{u_{w}(F, \Gamma), N-1\right\}+1,
$$

and further, we conjecture that

$$
u(F, \Gamma) \leq \max \left\{u_{w}(F, \Gamma), N-1\right\} .
$$

Here, we give a simple example satisfying $u_{w}(F, \Gamma)<u(F, \Gamma)$.

Example 1.14. Let $N>1$ be an integer. Let $(F, \Gamma)$ be a branched covering surface-knot $\left(S^{2}, \emptyset\right)+\sum_{i=1}^{N} h\left(\sigma_{i}, \sigma_{N+i}\right)$ of degree $2 N+1$. Then, $u_{w}(F, \Gamma)=0$ and $u(F, \Gamma)=N$.

Concerning Conjectures $1.12,1.13$ and the simplifying number and the weak simplifying number, we discuss in [10]. We also investigate concerning Conjecture 1.13 and the simplifying number and the weak simplifying number for branched covering surface-knots with black vertices in [11.

The paper is organized as follows. In Section 2, we review branched covering surface-knots and their chart presentations. In Section 3, we show Theorem 1.7. In Section 4, we show Theorem 1.8, In Section 5, we show Theorem 1.9 and Corollaries 1.10, 1.11.

\section{TERminology Change: BRANCHED COVERING SURFACE-KNOTS (FORMERLY 2-DIMENSIONAL BRAIDS) AND THEIR CHART PRESENTATIONS}

In this section, we review a branched covering surface-knot, formerly called a 2-dimensional braid over a surface-knot [8], which is an extended notion of 2-dimensional braids or surface braids over a 2-disk [3, 15, 13]. A branched covering surface-knot over a surface-knot $F$ is presented by a finite graph called a chart on a surface diagram of $F[8$ ] (see also [3, 5]). For two branched covering surface-knots of the same degree, they are equivalent if 
their surface diagrams with charts are related by a finite sequence of ambient isotopies of $\mathbb{R}^{3}$, and local moves called C-moves [3, 4, 5] and Roseman moves [8] (see also [12]).

Remark 2.1. We formerly called a branched covering surface-knot a 2dimensional braid over a surface-knot. The term "2-dimensional braid" is usually used for a surface properly embedded in a bidisk in the braid form over a 2-disk [5], and it has a boundary, but a branched covering surfaceknot is embedded in $\mathbb{R}^{4}$ and has no boundary. Hence, in order to avoid confusion, we change the notation.

We work in the smooth category. A surface-knot is the image of a smooth embedding of a closed surface into $\mathbb{R}^{4}$. Two surface-knots are equivalent if one is carried to the other by an ambient isotopy of $\mathbb{R}^{4}$.

2.1. Branched covering surface-knots over a surface-knot. Let $B^{2}$ be a 2-disk, and let $N$ be a positive integer. For a surface-knot $F$, let $N(F)=B^{2} \times F$ be a tubular neighborhood of $F$ in $\mathbb{R}^{4}$.

Definition 2.2. A closed surface $S$ embedded in $N(F)$ is called a branched covering surface-knot over $F$ of degree $N$ if it satisfies the followings.

(1) The restriction $\left.p\right|_{S}: S \rightarrow F$ is a branched covering map of degree $N$, where $p: N(F) \rightarrow F$ is the natural projection.

(2) The number of points consisting $S \cap p^{-1}(x)$ is $N$ or $N-1$ for any point $x \in F$.

Take a base point $x_{0}$ of $F$. Two branched covering surface-knots over $F$ of degree $N$ are equivalent if there is an ambient isotopy of $\mathbb{R}^{4}$ whose restriction to $N(F)=B^{2} \times F$ is a fiber-preserving ambient isotopy rel $p^{-1}\left(x_{0}\right)$ taking one to the other.

2.2. Chart presentation. Let $S$ be a branched covering surface-knot over a surface-knot $F$. A surface diagram of a surface-knot is the image of $F$ in $\mathbb{R}^{3}$ by a generic projection, equipped with the over/under information on sheets along each double point curve. In this paper, $F$ is an unknotted surface-knot in the standard form. We consider a surface diagram of $F$ as the image of $F$ by the projection $\mathbb{R}^{4} \rightarrow \mathbb{R}^{3},(x, y, z, t) \mapsto(x, y, z)$, which has no singularities. We identify the surface diagram of $F$ with $F$ itself, and we identify $N(F)$ with $I \times I \times F$, where the second $I$ is an interval in the fourth axis of $\mathbb{R}^{4}$.

We explain a chart on a surface diagram $F$. Consider the singular set $\operatorname{Sing}\left(p_{1}(S)\right)$ of the image of $S$ by the projection $p_{1}: I \times I \times F \rightarrow I \times F$, $(s, t, x) \mapsto(s, x)$. Perturbing $S$ if necessary, we assume that $\operatorname{Sing}\left(p_{1}(S)\right)$ consists of double point curves, triple points, and branch points. Moreover, we assume that the singularity set of the image of $\operatorname{Sing}\left(p_{1}(S)\right)$ by the projection to $F$ consists of a finite number of double points such that the preimages belong to double point curves of $\operatorname{Sing}\left(p_{1}(S)\right)$. Thus, the image of $\operatorname{Sing}\left(p_{1}(S)\right)$ by the projection to $F$ forms a finite graph $\Gamma$ on $F$ such that the degree of a vertex of $\Gamma$ is either 1,4 or 6 . An edge of $\Gamma$ corresponds to a double point curve, and a vertex of degree 1 (resp. 6) corresponds to a branch point (resp. a triple point). 
For such a graph $\Gamma$ obtained from a branched covering surface-knot $S$, we assign orientations and labels to edges of $\Gamma$ as follows. We consider an oriented path $\rho$ in $F$ such that $\rho \cap \Gamma$ is a point $x$ of an edge $e$ of $\Gamma$. Then, $F \cap p^{-1}(\rho)$ is a classical $N$-braid with one crossing in $p^{-1}(\rho)$ such that $x$ corresponds to the crossing of the $N$-braid, where $N$ is the degree of $S$. Let $\sigma_{i}^{\epsilon}(i \in\{1,2, \ldots, N-1\}, \epsilon \in\{+1,-1\})$ be the presentation of $S \cap p^{-1}(\rho)$. Then, assign $e$ the label $i$, and the orientation such that the normal vector of $\rho$ is coherent with (resp. opposite to) the orientation of $e$ if $\epsilon=+1$ (resp. $-1)$, where the normal vector of $\rho$ is the vector $\vec{n}$ such that $(\vec{v}(\rho), \vec{n})$ is the orientation of $F$ for a tangent vector $\vec{v}(\rho)$ of $\rho$ at $x$. In this situation, we say that an oriented path $\rho$ reads a braid $\sigma_{i}^{\epsilon}$. This is the chart of $S$.

For an unknotted surface-knot $F$ in the standard form, we define a chart on a surface diagram of $F$ as follows, see [5]. See [8] for a chart on a surface diagram which admits singularities.

Definition 2.3. Let $N$ be a positive integer. A finite graph $\Gamma$ on a surface diagram $F$ without singularities is called a chart of degree $N$ if it satisfies the following conditions.

(1) Every vertex has degree 1, 4, or 6.

(2) Every edge of $\Gamma$ is oriented and labeled by an element of $\{1,2, \ldots, N-1\}$ such that the adjacent edges around each of degree 1, 4, or 6 are oriented and labeled as shown in Fig. 3, where we depict a vertex of degree 1 by a black vertex, and a vertex of degree 6 by a white vertex, and we call a vertex of degree 4 a crossing.

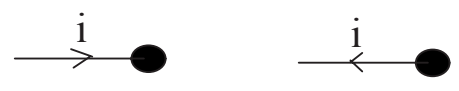

black vertex

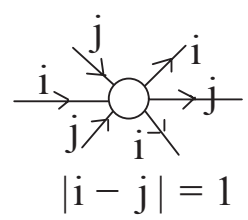

white vertex

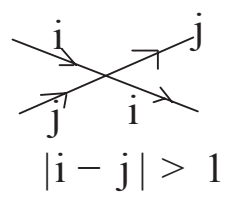

crossing

Figure 3. Vertices in a chart, where $i \in\{1, \ldots, N-1\}$. There are three types.

A black vertex (resp. a white vertex) of a chart corresponds to a branch point (resp. a triple point) of the branched covering surface-knot presented by the chart. We call an edge of a chart a chart edge or simply an edge. We regard diagonal edges connected with crossings as one edge with crossings, and we regard that a crossing is formed by a transverse intersection of two edges. A chart edge connected with no vertices or a chart edge with crossings is called a chart loop or simply a loop. A chart edge connected with two black vertices at endpoints is called a free edge. A chart is said to be empty if it is an empty graph.

Among six edges connected with a white vertex, we call an edge which is the middle of three adjacent edges with the coherent orientation a middle edge, and we call an edge which is not a middle edge a non-middle edge; see 
Fig. 4. For edges connected with a white vertex, we call a pair of edges separated by two edges at each side diagonal edges.

In this paper, for a white vertex $w$ such that the three adjacent edges oriented toward $w$ consist of two edges with the label $i$ and one edge with the label $j$, we call $w$ a white vertex of type $w_{i, j}$. For a crossing $c$ consisting of edges with the labels $i$ and $j$ such that the orientations of the edges form the right-handed orientation, we say $c$ a crossing of type $c_{i, j}$. See Fig. 4 .

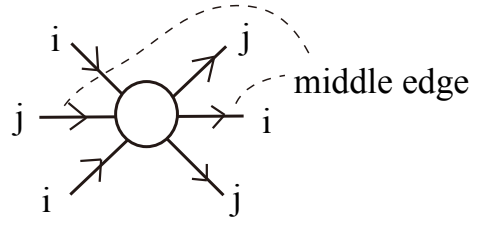

type wi,j

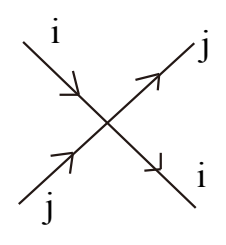

type $\mathrm{ci}, \mathrm{j}$

FIgURE 4. A white vertex of type $w_{i, j}$ and a crossing of type $c_{i, j}$.

A branched covering surface-knot over a surface-knot $F$ is presented by a chart $\Gamma$ on a surface diagram of $F[8]$. We present such a branched covering surface-knot by $(F, \Gamma)$.

2.3. C-moves. C-moves are local moves of a chart, consisting of three types: CI-moves, CII-moves, and CIII-moves. Let $\Gamma$ and $\Gamma^{\prime}$ be two charts of the same degree on a surface diagram $F$. We say $\Gamma$ and $\Gamma^{\prime}$ are related by a CI-move, CII-move or CIII-move if there exists a 2-disk $E$ in $F$ such that the loop $\partial E$ is in general position with respect to $\Gamma$ and $\Gamma^{\prime}$ and $\Gamma \cap(F-E)=\Gamma^{\prime} \cap(F-E)$, and the following conditions hold true.

(CI) There are no black vertices in $\Gamma \cap E$ nor $\Gamma^{\prime} \cap E$. There are 7 types called CI-M1-CI-M4 moves, and CI-R1-CI-R3 moves as in Fig. 5 .

(CII) $\Gamma \cap E$ and $\Gamma^{\prime} \cap E$ are as in Fig. 5, where $|i-j|>1$.

(CIII) $\Gamma \cap E$ and $\Gamma^{\prime} \cap E$ are as in Fig. 5, where $|i-j|=1$, and the black vertex is connected with a non-middle edge of a white vertex.

Let $\Gamma$ and $\Gamma^{\prime}$ be charts of the same degree on a surface diagram $F$. We say $\Gamma$ and $\Gamma^{\prime}$ are $C$-move equivalent if they are related by a finite sequence of C-moves and ambient isotopies of $F$ rel $x_{0}$, where $x_{0}$ is a base point of $F$. For charts $\Gamma$ and $\Gamma^{\prime}$, their presenting branched covering surface-knots are equivalent if the charts are C-move equivalent [3, 4, [5].

2.4. Roseman moves. Roseman moves for surface diagrams with charts of the same degree are defined by the original Roseman moves (see [12]) and moves for local surface diagrams with non-empty charts (see [8]), where we regard the diagrams for the original Roseman moves as equipped with empty charts.

For two surface diagrams with charts of the same degree, their presenting branched covering surface-knots are equivalent if they are related by a finite sequence of ambient isotopies of $\mathbb{R}^{3}$ and Roseman moves for surface diagrams with charts [8]. 


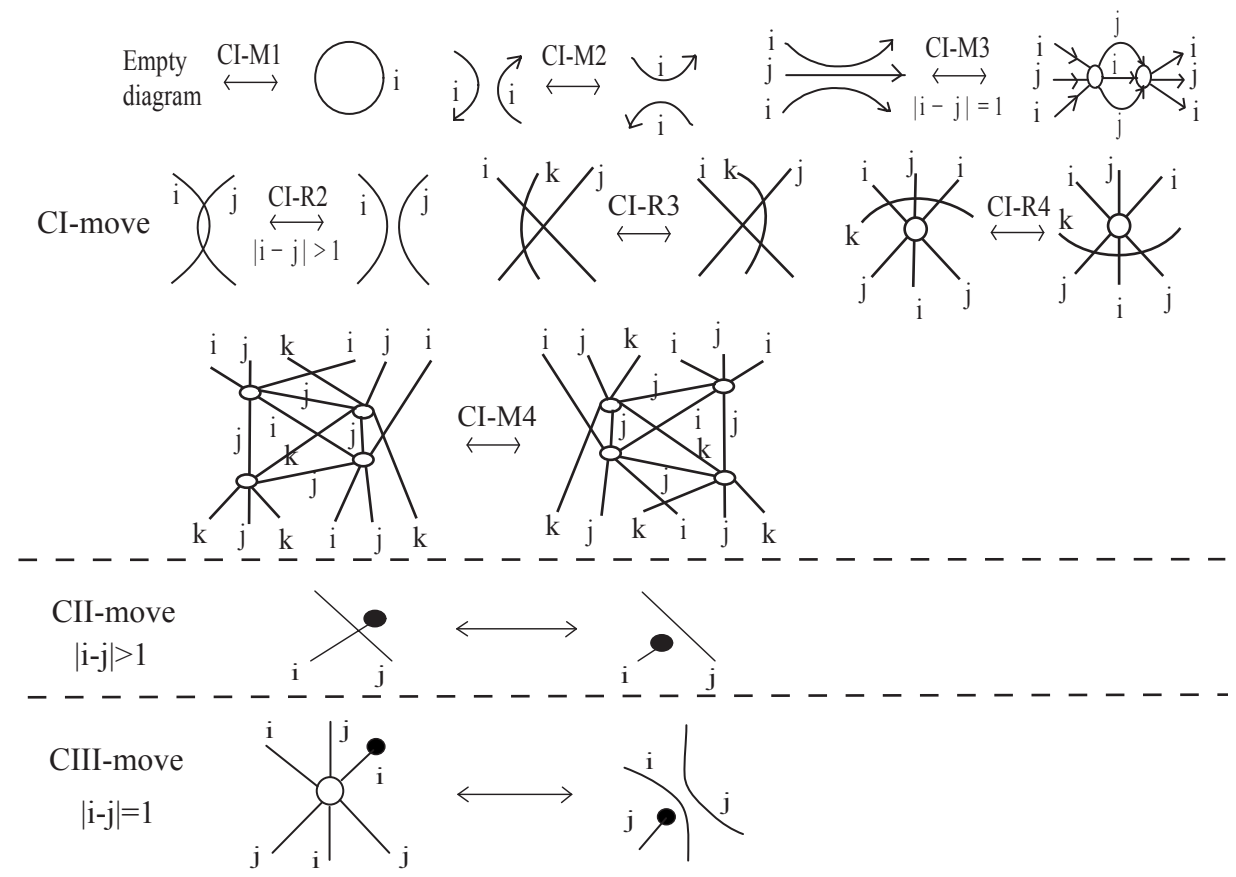

Figure 5. C-moves. For simplicity, we omit orientations of some edges.

\section{Simplifying BRANCHED COVERING SURFACE-KNOTS}

We denote by $h(a, b)$ for braids $a, b$ a 1-handle equipped with a chart without black vertices such that the cocore (resp. the orientation-reversed core loop) reads the presentation of $a$ (resp. $b$ ), and we assume that $h(a, b)$ is well-defined, that is, $a$ and $b$ commute. Unless otherwise said, we assume that 1-handles are attached to a fixed 2-disk $E$ in $F$ such that in $E$ there are no chart edges nor vertices except those of 1-handles. For 1-handles with charts $h\left(a_{1}, b_{1}\right), \ldots, h\left(a_{g}, b_{g}\right)$, we denote by $\sum_{i=1}^{g} h\left(a_{i}, b_{i}\right)$ the result of the 1-handle addition. We say 1-handles with charts are equivalent if their presenting branched covering surface-knots are equivalent relative to a tubular neighborhood of $F-E$, and we use the notation " $\sim$ " to denote equivalence relation. Let $N$ be the degree of branched covering surfaceknots.

Lemma 3.1. For $N$-braids $a$ and $b$, we have

$$
h(a, b) \sim h\left(a^{-1}, b^{-1}\right) .
$$

In particular,

$$
h\left(\sigma_{i}, e\right) \sim h\left(\sigma_{i}^{-1}, e\right),
$$

where $i \in\{1, \ldots, N-1\}$.

Proof. By rotating the 1-handle around and taking the orientation reversal of the original core loop as the new core loop, we have the result (see Fig. 6). See [9, Lemma 4.1]. 


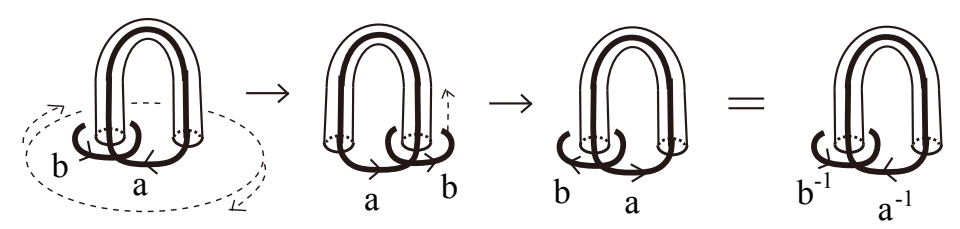

FIgURE 6 . The image of $h(a, b) \sim h\left(a^{-1}, b^{-1}\right)$, where actually the chart consists of chart edges, white vertices and crossings.

Lemma 3.2. Together with $\sum_{i=1}^{N-1} h\left(\sigma_{i}, e\right)$, a 1-handle $h(e, e)$ transforms to $h(e, b)$ for any braid $b$ :

$$
\sum_{i=1}^{N-1} h\left(\sigma_{i}, e\right)+h(e, e) \sim \sum_{i=1}^{N-1} h\left(\sigma_{i}, e\right)+h(e, b) .
$$

Proof. Let $c$ be a braid. By moving an end of $h(e, c)$ between the ends of $h\left(\sigma_{i}, e\right)$ and applying a CI-move as indicated in Fig. 7, $h(e, c)$ changes to $h\left(e, c \sigma_{i}^{\epsilon}\right)(\epsilon=-1)$, and $h\left(\sigma_{i}, e\right)$ is unchanged. See also [9, Lemma 4.6]. The sign $\epsilon$ changes to +1 by changing the orientation of the chart loop of $h\left(\sigma_{i}, e\right)$ by rotating it around to be $h\left(\sigma_{i}^{-1}, e\right)$. Hence, $h(e, c)+h\left(\sigma_{i}, e\right) \sim$ $h\left(e, c \sigma_{i}^{\epsilon}\right)+h\left(\sigma_{i}, e\right)$, where $\epsilon \in\{+1,-1\}$. Since $\sum_{i=1}^{N-1} h\left(\sigma_{i}, e\right)$ covers all labels $1, \ldots, N-1$, by repeating this process several times to make chart loops surrounding the cocore of the first 1-handle such that the loops present the given braid $b$, we have the required result.

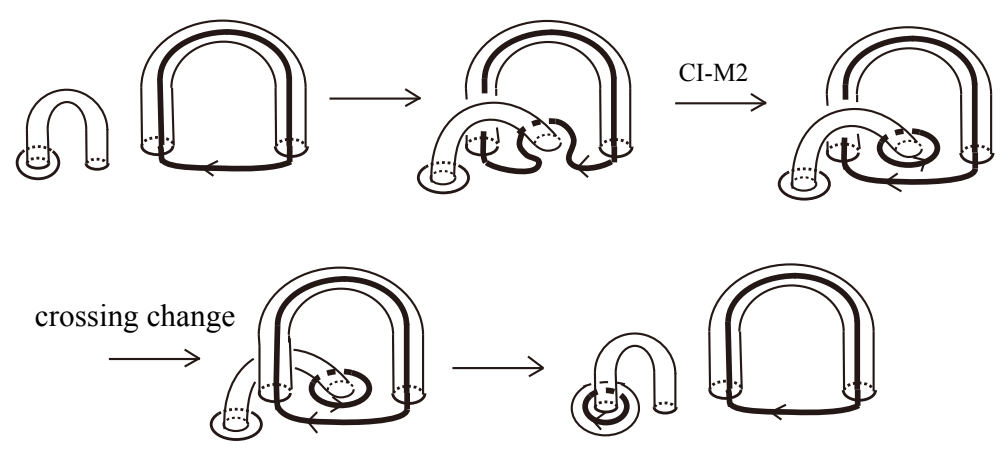

FiguRE 7. $h(e, c)+h\left(\sigma_{i}, e\right) \sim h\left(e, c \sigma_{i}^{\epsilon}\right)+h\left(\sigma_{i}, e\right)$. The orientations of chart loops are for the case $\epsilon=-1$. For simplicity, we omit the label of chart loops, and the part presenting $c$ is an image.

Lemma 3.3. Together with $\sum_{i=1}^{N-1} h\left(\sigma_{i}, e\right)$, a 1-handle $h(e, e)$ transforms to a 1-handle with an empty chart such that each end is attached to anywhere.

Proof. Let $E$ be the 2-disk where $\sum_{i=1}^{N-1} h\left(\sigma_{i}, e\right)$ and $h=h(e, e)$ are attached. Let $E_{1}$ and $E_{2}$ be 2-disks where we want to move the ends of $h$. Consider oriented paths from $E$ to $E_{i}(i=1,2)$ such that the intersection with the 
chart consists of the transverse intersections with chart edges. Let $b_{1}$ and $b_{2}$ be the braids presented by these paths, respectively. Then, by Lemma 3.2 by using $\sum_{i=1}^{N-1} h\left(\sigma_{i}, e\right)$, we transform $h$ to $h\left(e, b_{1}^{-1} b_{2}\right)$. We move the ends to $E_{1}$ and $E_{2}$ along the paths, and then $h\left(e, b_{1}^{-1} b_{2}\right)$ transforms to a 1handle with chart loops surrounding the cocore presenting $b_{1}\left(b_{1}^{-1} b_{2}\right) b_{2}^{-1} \sim e$, which is equivalent to a 1-handle with an empty chart. In other words, by applying C1-M2 moves while moving the ends of $h$, we eliminate chart loops surrounding the cocore. See Fig. 8 .

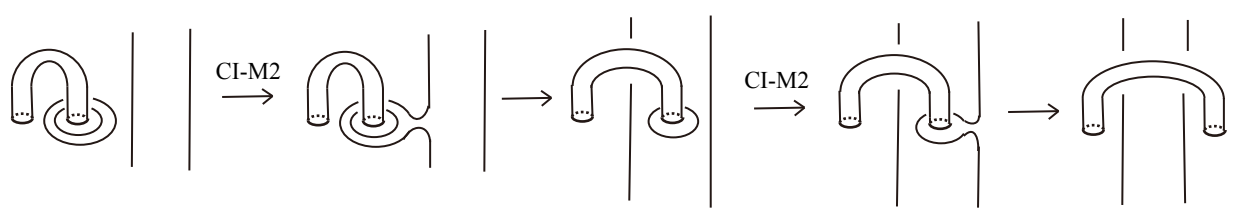

Figure 8. Moving an end of a 1-handle to make a bridge. For simplicity, we omit labels and orientations of chart edges.

Let $E_{1}, E_{2}$ be 2-disks such that we have a 1-handle with an empty chart whose ends are attached to $E_{1}$ and $E_{2}$, respectively. We say that we have a bridge between $E_{1}$ and $E_{2}$.

Lemma 3.4. Let $\rho$ be a chart loop with the label $i$ which has no crossings. Then, $\rho$ is eliminated by an addition of $h\left(\sigma_{i}, b\right)$ to a neighborhood of $\rho$, where $b$ is a braid, and the 1-handle is unchanged. In particular, we have

$$
h\left(\sigma_{i}, b\right)+h\left(\sigma_{i}, e\right) \sim h\left(\sigma_{i}, b\right)+h(e, e)
$$

and

$$
h\left(\sigma_{i}, b\right)+h\left(e, \sigma_{i}^{\epsilon}\right) \sim h\left(\sigma_{i}, b\right)+h(e, e),
$$

where $i \in\{1, \ldots, N-1\}, \epsilon \in\{+1,-1\}$ and $b$ is a braid.

Proof. By applying a CI-M2 move between the edges of the label $i$ of $\rho$ and $h\left(\sigma_{i}, b\right)$, and sliding an end of the 1-handle, we eliminate the chart loop $\rho$, and the 1-handle is unchanged. The relation (3.4) is shown by taking $\rho$ as the chart loop along the core loop of the second 1-handle $h\left(\sigma_{i}, e\right)$ (see Fig. 9 , and the relation (3.5) is shown by taking $\rho$ as the chart loop surrounding the cocore of the second 1-handle $h\left(e, \sigma_{i}^{\epsilon}\right)$.

We remark that by repeating the inverse process of (3.5) for $b=e$, $h\left(\sigma_{i}, e\right)+h(e, e) \sim h\left(\sigma_{i}, e\right)+h\left(e, \sigma_{i}^{\epsilon}\right)$ several times, we have Lemma 3.2

Lemma 3.5. Let $\rho$ be a chart loop with the label $i$ with crossings such that $\rho$ as a closed path reads a braid $c$. Then, $\rho$ is eliminated by an addition of $h\left(\sigma_{i}, b\right)$ to a neighborhood of the initial point of $\rho$, where $b$ is a braid, and the 1-handle changes to $h\left(\sigma_{i}, b c^{-1}\right)$. In particular, we have

$$
h\left(\sigma_{i}, b\right)+h\left(\sigma_{i}, c\right) \sim h\left(\sigma_{i}, b c\right)+h(e, c),
$$

and

$$
h\left(\sigma_{i}, b\right)+h\left(c, \sigma_{i}^{\epsilon}\right) \sim h\left(\sigma_{i}, b c^{-\epsilon}\right)+h(c, e),
$$




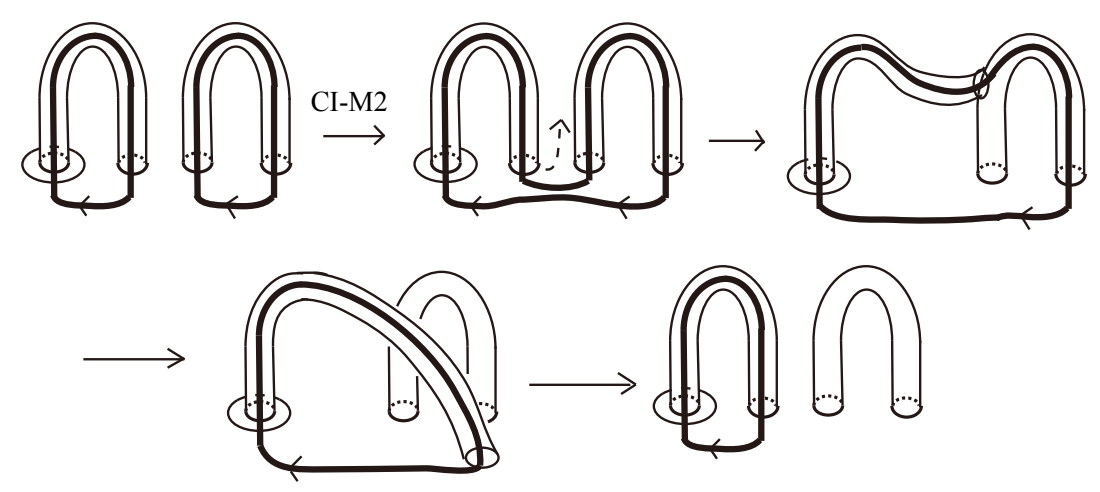

FiguRE 9. $h\left(\sigma_{i}, b\right)+h\left(\sigma_{i}, e\right) \sim h\left(\sigma_{i}, b\right)+h(e, e)$. We omit the label of chart loops, and the part presenting $b$ is an image.

where $i \in\{1, \ldots, N-1\}, \epsilon \in\{+1,-1\}$ and $b, c$ are braids such that the 1-handles have only crossings for vertices.

Proof. Similar to the proof of Lemma 3.4, by applying a CI-M2 move and sliding an end of the 1-handle, we eliminate the chart loop $\rho$. When the 1-handle slides across a crossing, the crossing is collected on the 1-handle. It follows that when the moving end comes back to a neighborhood of the other end, the resulting 1-handle is $h\left(\sigma_{i}, b c^{-1}\right)$. See Fig. 10. The relation (3.6) is shown by taking $\rho$ as the chart loop around the core loop of the second 1-handle $h\left(\sigma_{i}, e\right)$. Since the braid $c$ of $h\left(\sigma_{i}, c\right)$ is read by the orientation-reversed core loop, the closed path $\rho$ reads $c^{-1}$ and the first 1handle becomes $h\left(\sigma_{i}, b c\right)$. The relation (3.7) is shown by taking $\rho$ as the chart loop surrounding the cocore of the second 1-handle $h\left(e, \sigma_{i}^{\epsilon}\right)$. Since the braid $c$ of $h\left(c, \sigma_{i}^{\epsilon}\right)$ is read by the core loop, $\rho$ reads $c$ and the first 1-handle becomes $h\left(\sigma_{i}, b c^{-\epsilon}\right)$. See also [9, Figs. 18, 19 and Lemma 6.7].

Proof of Theorem 1.7. We show the second inequality (1.6). If $b(\Gamma)=0$, then, by Lemma 3.6, white vertices consist of several pairs such that the types of each pair is $\left\{w_{i, j}, w_{j, i}\right\}$. By Lemma 3.3 , by an addition of 1 -handles $\sum_{i=1}^{N-1} h\left(\sigma_{i}, e\right)+h(e, e)$, we transform the 1-handle $h(e, e)$ to make a bridge between neighborhoods of a pair of white vertices of types $\left\{w_{i, j}, w_{j, i}\right\}$. Then, by an ambient isotopy and C1-moves, we eliminate the pair of white vertices as indicated in Fig. 11. Then, $\sum_{i=1}^{N-1} h\left(\sigma_{i}, e\right)$ is unchanged. By adding $h(e, e)$ and applying this process to every pair of types $\left\{w_{i, j}, w_{j, i}\right\}$, we deform $(F, \Gamma)$ to a branched covering surface-knot $\left(F^{\prime}, \Gamma^{\prime}\right)+\sum_{i=1}^{N-1} h\left(\sigma_{i}, e\right)$, where $F^{\prime}=F+\sum_{j=1}^{n} h$ for a non-negative integer $n$ and $\Gamma^{\prime}$ is a chart without white vertices. Since we obtained $\left(F^{\prime}, \Gamma^{\prime}\right)+\sum_{i=1}^{N-1} h\left(\sigma_{i}, e\right)$ from $(F, \Gamma)$ by an addition of $\sum_{i=1}^{N-1} h\left(\sigma_{i}, e\right)$ and $w(\Gamma) / 2$ copies of $h(e, e)$, we see that $n=$ $w(\Gamma) / 2$.

The chart $\Gamma^{\prime}$ consists of several loops with $c(\Gamma)$ number of crossings. By Lemmas 3.4 and 3.5 , by using $\sum_{i=1}^{N-1} h\left(\sigma_{i}, e\right)$, we eliminate loops. The result is $(F, \emptyset)$ with $w(\Gamma) / 2$ copies of $h(e, e)$ and $\sum_{i=1}^{N-1} h\left(\sigma_{i}, b_{i}\right)$ for some braids $b_{i}$ such that vertices are only crossings on $\sum_{i=1}^{N-1} h\left(\sigma_{i}, b_{i}\right)$. Then add several 


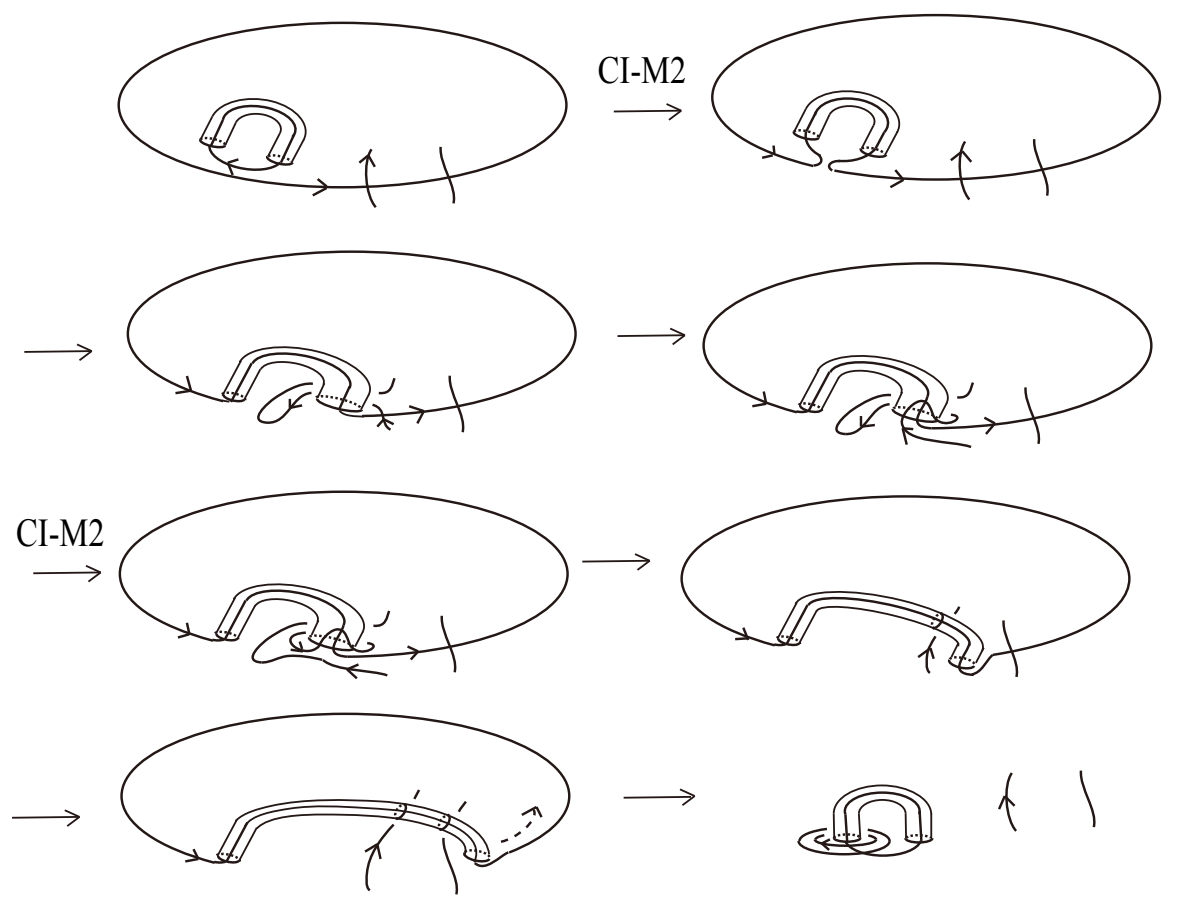

Figure 10. Collecting crossings on a 1-handle. For simplicity, we omit labels of chart edges.

copies of $h(e, e)$ so that we have $c(\Gamma)$ copies of $h(e, e)$. Each crossing consists of a chart loop along the core loop and a chart loop $\rho$ parallel to the cocore of a 1-handle such that there is only one crossing on $\rho$. Let $E$ be the 2-disk where 1-handles are attached. Let $c$ be a crossing such that there exists a path from $E$ to $c$ which does not intersect with chart edges nor vertices. For such a crossing $c$, let $j$ be the label of the consisting loop $\rho$ parallel to the cocore. By the inverse process of Lemma 3.4 by using $h\left(\sigma_{j}, b_{j}\right)$, we change a 1-handle $h(e, e)$ to $h\left(\sigma_{j}, e\right)$. By an ambient isotopy, we move $h=h\left(\sigma_{j}, e\right)$ to a neighborhood of $c$, and by Lemma 3.5, we apply a CI-M2 move and slide an end of the 1-handle $h$ along $\rho$. Thus we collect the crossing $c$ on $h$, and then $h$ transforms to the form $h\left(\sigma_{j}, \sigma_{k}^{\epsilon}\right)(|j-k|>1, \epsilon \in\{+1,-1\})$, attached on $E$. By repeating this process, we collect each crossing on a 1-handle, and thus we have 1-handles in the form $h\left(\sigma_{j}, \sigma_{k}^{\epsilon}\right)(|j-k|>1, \epsilon \in\{+1,-1\})$, and $\sum_{i=1}^{N-1} h\left(\sigma_{i}, e\right)$, and $\max \{0, w(\Gamma) / 2-c(\Gamma)\}$ copies of 1-handles $h(e, e)$, and we have a weak simplified form. Thus we have the second inequality $u_{w}(F, \Gamma) \leq \max \{w(\Gamma) / 2, c(\Gamma)\}+N-1$.

If $b(\Gamma)>0$ and $\Gamma$ has black vertices, then, by Lemma 3.7, the number of edges connected with black vertices with the orientation from a black vertex equals that with the orientation toward a black vertex. Since an edge with the label $i$ connected with a black vertex changes to an edge with the label $j(|i-j|=1)$ connected with a black vertex by increasing one white vertex as in Fig. 12 , by increasing at most $(b(\Gamma) / 2)(N-2)$ white vertices, we deform $\Gamma$ so that the number of edges connected with 
black vertices with the orientation from a black vertex and with the label $i$ equals that with the orientation toward a black vertex and with the label $i$. By considering the chart obtained from $\Gamma$ by making bridges by 1-handles between neighborhoods of black vertices, and eliminating black vertices and joining the edges formerly connected with them through 1-handles forming bridges, by Lemma 3.6 we see that for this new chart and hence also $\Gamma$, the number of white vertices of type $w_{i, j}$ equals that of type $w_{j, i}$. Thus, by the same argument as in the case $b(\Gamma)=0$, we see that by an addition of $\sum_{i=1}^{N-1} h\left(\sigma_{i}, e\right)$ and $\lfloor\{w(\Gamma)+(b(\Gamma) / 2)(N-2)\} / 2\rfloor=\lfloor w(\Gamma) / 2+(b(\Gamma) / 4)(N-$ $2)\rfloor$ copies of $h(e, e)$, we deform the union of $\Gamma$ and the charts on 1-handles to a chart without white vertices and moreover we have $\sum_{i=1}^{N-1} h\left(\sigma_{i}, e\right)$. The rest of the argument is the same as the case $b(\Gamma)=0$ : we eliminate chart loops by using $\sum_{i=1}^{N-1} h\left(\sigma_{i}, e\right)$, and by an addition of 1-handles $h(e, e)$ we move each crossing to be on one 1-handle. By applying CII-moves if necessary, black vertices become the endpoints of free edges, and the resulting chart consists of several free edges and 1-handles with chart loops; thus we have the weak simplified form and we have the first inequality (1.5): $u_{w}(F, \Gamma) \leq$ $\max \{\lfloor w(\Gamma) / 2+(b(\Gamma) / 4)(N-2)\rfloor, c(\Gamma)\}+N-1$.

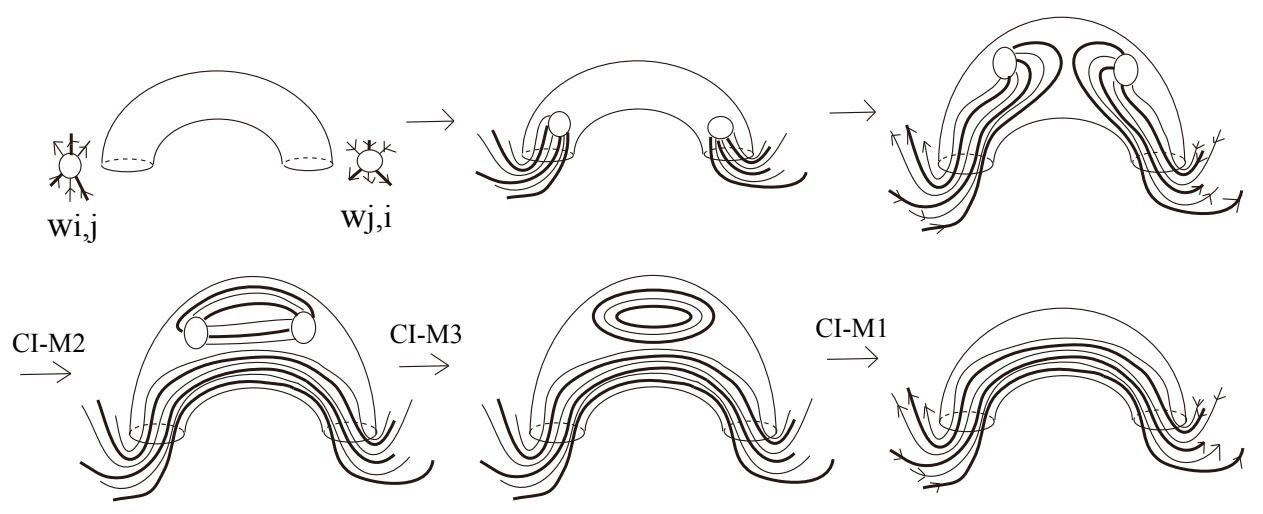

FigURE 11. Elimination of a pair of white vertices $w_{i, j}$ and $w_{j, i}$. We omit labels and orientations of chart edges.

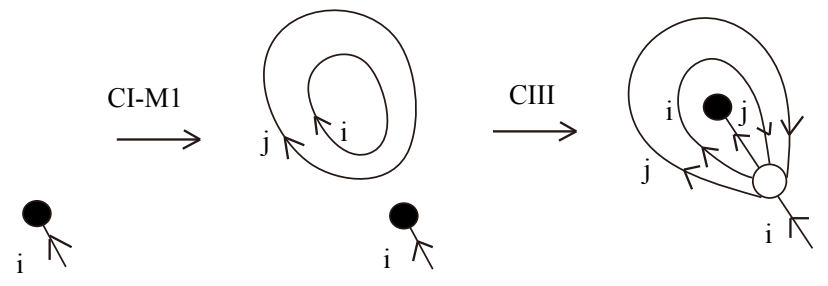

FIGURE 12. Changing the label of the chart edge connected with a black vertex. The orientations of chart edges are an example. 
Lemma 3.6. Let $\Gamma$ be a chart of degree $N$ such that $b(\Gamma)=0$, where $b(\Gamma)$ is the number of black vertices. Then, the number of white vertices of type $w_{i, j}$ equals that of type $w_{j, i}$, where $|i-j|=1, i, j \in\{1, \ldots, N-1\}$.

Proof. In the set of edges connected with white vertices, an edge with the label 1 appears only as an edge connected with a white vertex of type $w_{1,2}$ or $w_{2,1}$. Around a white vertex $w$ of type $w_{1,2}$ (resp. $w_{2,1}$ ), the number of edges with the label 1 with the orientation toward $w$ is 2 (resp. 1), and the number of those with the orientation from $w$ is 1 (resp. 2). Since there are no black vertices, the number of edges around white vertices with the label 1 and the orientation toward the vertex equals that with the orientation from the vertex, where we count an edge twice if each of its endpoint is connected with a white vertex. It follows that the number of white vertices of type $w_{1,2}$ equals that of type $w_{2,1}$. Thus, repeating the same argument for edges of label $i$ for $i=2,3, \ldots, N-2$ in this order, we obtain the required result.

Lemma 3.7. For a chart, the number of edges connected with black vertices with the orientation from a black vertex equals that with the orientation toward a black vertex.

Proof. We see the result from the fact that around a crossing/white vertex, the number of the edges with the orientation from the crossing/white vertex equals that with the orientation toward it.

\section{TO MORE SIMPLIFIED FORMS I}

In this section, we show Theorem 1.8, Let $N$ be the degree of branched covering surface-knots.

Lemma 4.1. We have

$$
h\left(\sigma_{j}, b_{j}\right)+h\left(\sigma_{k}, b_{k}\right)+h\left(\sigma_{j}, \sigma_{k}^{\epsilon}\right) \sim h\left(\sigma_{j}, b_{j}\right)+h\left(\sigma_{k}, b_{k}\right)+h\left(\sigma_{k}, \sigma_{j}^{-\epsilon}\right),
$$

where $|j-k|>1(j, k \in\{1, \ldots, N-1\}), \epsilon \in\{+1,-1\}$, and $b_{j}$ and $b_{k}$ are braids.

Proof. Assume that we have $h\left(\sigma_{j}, b_{j}\right)+h\left(\sigma_{k}, b_{k}\right)+h\left(\sigma_{j}, \sigma_{k}^{\epsilon}\right)$. Put $h_{1}=$ $h\left(\sigma_{j}, b_{j}\right), h_{2}=h\left(\sigma_{k}, b_{k}\right)$, and $h_{3}=h\left(\sigma_{j}, \sigma_{k}^{\epsilon}\right)$. By Lemma 3.5, by applying a CI-M2 move to the edges with the label $j$ of $h_{1}$ and $h_{3}$, and sliding an end of $h_{1}$ along $h_{3}, h_{1}$ becomes $h\left(\sigma_{j}, b_{j} \sigma_{k}^{\epsilon}\right)$ and $h_{3}$ becomes $h\left(e, \sigma_{k}^{\epsilon}\right)$, and we have

$$
h\left(\sigma_{j}, b_{j} \sigma_{k}^{\epsilon}\right)+h\left(\sigma_{k}, b_{k}\right)+h\left(e, \sigma_{k}^{\epsilon}\right) .
$$

By applying Lemma 3.5 to $h_{3}=h\left(e, \sigma_{k}^{\epsilon}\right)$ and $h_{2}=h\left(\sigma_{k}, b_{k}\right), h_{3}$ becomes $h(e, e)$, and $h_{2}$ is unchanged. We have

$$
h\left(\sigma_{j}, b_{j} \sigma_{k}^{\epsilon}\right)+h\left(\sigma_{k}, b_{k}\right)+h(e, e) .
$$

By applying the inverse process of Lemma 3.5 to $h_{2}=h\left(\sigma_{k}, b_{k}\right)$ and $h_{3}=$ $h(e, e), h_{3}$ becomes $h\left(\sigma_{k}, e\right)$ and $h_{2}$ is unchanged, and we have

$$
h\left(\sigma_{j}, b_{j} \sigma_{k}^{\epsilon}\right)+h\left(\sigma_{k}, b_{k}\right)+h\left(\sigma_{k}, e\right) .
$$

By applying Lemma 3.5 to $h_{1}=h\left(\sigma_{j}, b_{j} \sigma_{k}^{\epsilon}\right)$ and $h_{3}=h\left(\sigma_{k}, e\right)$, by applying a CI-M2 move to the edges with the label $k$ and collecting a crossing on $h_{3}$, $h_{3}$ becomes $h\left(\sigma_{k}, \sigma_{j}^{-\epsilon}\right)$ and $h_{1}$ becomes $h\left(\sigma_{j}, b_{j}\right)$, and we have

$$
h\left(\sigma_{j}, b_{j}\right)+h\left(\sigma_{k}, b_{k}\right)+h\left(\sigma_{k}, \sigma_{j}^{-\epsilon}\right),
$$


which is the required result.

Lemma 4.2. For $j<k$ with $|j-k|>1$,

$$
\sum_{i=1}^{N-1} h\left(\sigma_{i}, b_{i}\right)+h\left(\sigma_{j}, \sigma_{k}^{\epsilon}\right) \sim \sum_{i=1}^{N-1} h\left(\sigma_{i}, b_{i}\right)+h\left(\sigma_{1}, \sigma_{3}^{\epsilon}\right),
$$

and for $j>k$ with $|j-k|>1$,

$$
\sum_{i=1}^{N-1} h\left(\sigma_{i}, b_{i}\right)+h\left(\sigma_{j}, \sigma_{k}^{\epsilon}\right) \sim \sum_{i=1}^{N-1} h\left(\sigma_{i}, b_{i}\right)+h\left(\sigma_{1}, \sigma_{3}^{-\epsilon}\right),
$$

where $j, k \in\{1, \ldots, N-1\}, \epsilon \in\{+1,-1\}$, and $b_{i}$ are braids.

Proof. It suffices to show that for $j, k$ with $|j-k|>1$ and $|j-k+1|>1$,

$$
\sum_{i=1}^{N-1} h\left(\sigma_{i}, b_{i}\right)+h\left(\sigma_{j}, \sigma_{k}^{\epsilon}\right) \sim \sum_{i=1}^{N-1} h\left(\sigma_{i}, b_{i}\right)+h\left(\sigma_{j}, \sigma_{k-1}^{\epsilon}\right)
$$

and for $j, k$ with $|j-k|>1$ and $|j-k-1|>1$,

$$
\sum_{i=1}^{N-1} h\left(\sigma_{i}, b_{i}\right)+h\left(\sigma_{j}, \sigma_{k}^{\epsilon}\right) \sim \sum_{i=1}^{N-1} h\left(\sigma_{i}, b_{i}\right)+h\left(\sigma_{j-1}, \sigma_{k}^{\epsilon}\right) .
$$

The relation (4.2) follows from (4.4) and 4.5). Since $h\left(\sigma_{3}, \sigma_{1}^{\epsilon}\right) \sim h\left(\sigma_{1}, \sigma_{3}^{-\epsilon}\right)$ by Lemma 4.1, (4.3) follows from (4.4) and (4.5).

We show (4.4). Since $h\left(\sigma_{k-1}, b_{k-1}\right) \sim h\left(\sigma_{k-1}, \sigma_{j}^{-\epsilon} \sigma_{j}^{\epsilon} b_{k-1}\right)$ and $h\left(\sigma_{k}, b_{k}\right) \sim$ $h\left(\sigma_{k}, \sigma_{j}^{-\epsilon} \sigma_{j}^{\epsilon} b_{k}\right)$, by Lemma 5.2 or Lemma 3.5 . by sliding $h\left(\sigma_{j}, \sigma_{k}^{\epsilon}\right)$ along the cocores and collecting the crossings, $\sum_{i=1}^{N-1} h\left(\sigma_{i}, b_{i}\right)+h\left(\sigma_{j}, \sigma_{k}^{\epsilon}\right)$ deforms to

$$
\sum_{i=1}^{N-1} h\left(\sigma_{i}, b_{i}\right)+h\left(\sigma_{j}, \sigma_{k}^{\epsilon} \sigma_{k-1}^{\epsilon} \sigma_{k}^{\epsilon} \sigma_{k-1}^{-\epsilon} \sigma_{k}^{-\epsilon}\right) .
$$

Since $\sigma_{k}^{\epsilon} \sigma_{k-1}^{\epsilon} \sigma_{k}^{\epsilon} \sigma_{k-1}^{-\epsilon} \sigma_{k}^{-\epsilon} \sim\left(\sigma_{k}^{\epsilon} \sigma_{k-1}^{\epsilon} \sigma_{k}^{\epsilon} \sigma_{k-1}^{-\epsilon} \sigma_{k}^{-\epsilon} \sigma_{k-1}^{-\epsilon}\right) \sigma_{k-1}^{\epsilon} \sim e \sigma_{k-1}^{\epsilon} \sim \sigma_{k-1}^{\epsilon}$, we have

$$
\sum_{i=1}^{N-1} h\left(\sigma_{i}, b_{i}\right)+h\left(\sigma_{j}, \sigma_{k-1}^{\epsilon}\right) .
$$

Thus, we have (4.4). The second relation 4.5$)$ is obtained by using Lemma 4.1 and 4.4 as follows. By Lemma 4.1. $\sum_{i=1}^{N-1} h\left(\sigma_{i}, b_{i}\right)+h\left(\sigma_{j}, \sigma_{k}^{\epsilon}\right) \sim$ $\sum_{i=1}^{N-1} h\left(\sigma_{i}, b_{i}\right)+h\left(\sigma_{k}, \sigma_{j}^{-\epsilon}\right)$. By 4.4), this is equivalent to $\sum_{i=1}^{N-1} h\left(\sigma_{i}, b_{i}\right)+$ $h\left(\sigma_{k}, \sigma_{j-1}^{-\epsilon}\right)$. Since $h\left(\sigma_{k}, \sigma_{j-1}^{-\epsilon}\right) \sim h\left(\sigma_{j-1}, \sigma_{k}^{\epsilon}\right)$, we have 4.5$)$.

Proof of Theorem 1.8. By Lemma 3.5. $h\left(\sigma_{1}, \sigma_{3}^{-1}\right)+h\left(\sigma_{1}, \sigma_{3}\right)$ is equivalent to $h\left(\sigma_{1}, \sigma_{3}^{-1} \sigma_{3}\right)+h\left(e, \sigma_{3}\right)$, which is equivalent to $h\left(\sigma_{1}, e\right)+h\left(e, \sigma_{3}\right)$. These deform to $h(e, e)+h(e, e)$ by an addition of $\sum_{i=1}^{N-1} h\left(\sigma_{i}, e\right)$. Hence, by this argument and Theorem 1.2 and Lemma 4.2 , by an addition of finitely many 1-handles in the form $h\left(\sigma_{i}, e\right)$, or $h(e, e)(i \in\{1, \ldots, N-1\})$, to appropriate places in $F,(F, \Gamma)$ deforms to

$$
\left(F, \Gamma_{0}\right)+\sum_{i=1}^{N-1} h\left(\sigma_{i}, e\right)+\sum h\left(\sigma_{1}, \sigma_{3}^{\epsilon}\right)+\sum h(e, e),
$$


where $\epsilon$ is either +1 or -1 .

\section{TO MORE SIMPLIFIED FORMS II}

In this section, we show Theorem 1.9 and Corollaries 1.10, 1.11, Let $N$ be the degree of branched covering surface-knots.

Lemma 5.1. Together with $\sum_{i=1}^{N-1} h\left(\sigma_{i}, b_{i}\right)$, a 1-handle $h(a, b)$ moves anywhere, where $b_{i}, a, b$ are braids.

Proof. By moving the whole 1-handle instead of an end of the 1-handle as in the proofs of Lemmas 3.2 and 3.3, we have the required result. See Fig. 13 .

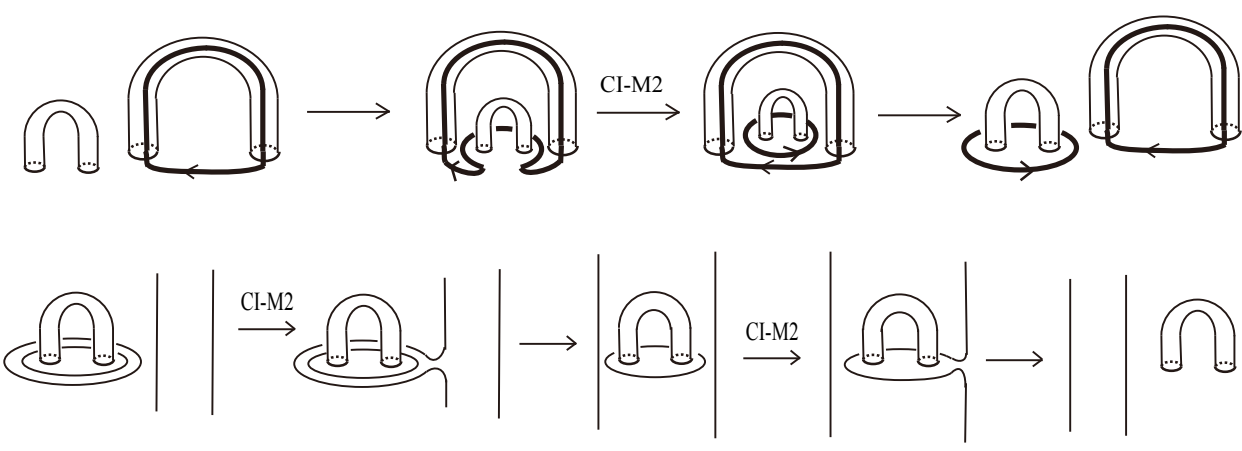

Figure 13. Moving a 1-handle to make a chart loop surrounding it (upper figures), and moving a 1-handle through chart edges (lower figures). The orientations of chart loops are an example. For simplicity, we omit labels and orientations of some chart edges.

Lemma 5.2. We have

$\sum_{i \neq j} h\left(\sigma_{i}, b_{i}\right)+h\left(\sigma_{j}, b \sigma_{k}^{\epsilon} b^{\prime}\right)+h\left(\sigma_{k}, c\right) \sim \sum_{i \neq j} h\left(\sigma_{i}, b_{i}\right)+h\left(\sigma_{j}, b b^{\prime}\right)+h\left(\sigma_{k}, c \sigma_{j}^{-\epsilon}\right)$, where $|j-k|>1, \epsilon \in\{+1,-1\}$, and $b_{i}, b, b^{\prime}, c$ are braids such that the 1-handles have only crossings as vertices.

Proof. Assume that we have $\sum_{i \neq j} h\left(\sigma_{i}, b_{i}\right)+h\left(\sigma_{j}, b \sigma_{k}^{\epsilon} b^{\prime}\right)+h\left(\sigma_{k}, c\right)$. By Lemma 5.1, we move $h=h\left(\sigma_{k}, c\right)$ to a neighborhood $E$ of the chart loop $\rho$ with the label $k$ parallel to the cocore of $h^{\prime}=h\left(\sigma_{j}, b \sigma_{k}^{\epsilon} b^{\prime}\right)$ presenting $\sigma_{k}$ of $b \sigma_{k}^{\epsilon} b^{\prime}$. Then, by Lemma 3.5, by applying a CI-M2 move and sliding an end of $h$ along $\rho$, we eliminate $\rho$ to collect on $h$ the crossing formed by $\rho$ and the chart loop along the core loop of $h^{\prime}$ with the label $j$. The 1handle $h$ becomes $h\left(\sigma_{k}, c \sigma_{j}^{-\epsilon}\right)$ attached to $E$, and $h^{\prime}$ becomes $h\left(\sigma_{j}, b b^{\prime}\right)$. By CI-M2 moves, when $h$ comes back to the original place, it is surrounded by chart loops presenting $b$. We eliminate these loops by Lemma 3.4. by using the other 1-handles $\sum_{i \neq j} h\left(\sigma_{i}, b_{i}\right)+h\left(\sigma_{j}, b b^{\prime}\right)$. Then, we have $\sum_{i \neq j} h\left(\sigma_{i}, b_{i}\right)+h\left(\sigma_{j}, b b^{\prime}\right)+h\left(\sigma_{k}, c \sigma_{j}^{-\epsilon}\right)$, which is the required result. 
Lemma 5.3. We have

$\sum_{i \neq j} h\left(\sigma_{i}, b_{i}\right)+h\left(\sigma_{j}, b \sigma_{k}^{\epsilon} b^{\prime}\right)+h\left(\sigma_{j}, c\right) \sim \sum_{i \neq j} h\left(\sigma_{i}, b_{i}\right)+h\left(\sigma_{j}, b b^{\prime}\right)+h\left(\sigma_{j}, c \sigma_{k}^{\epsilon}\right)$, where $|j-k|>1, b_{i}, b, b^{\prime}, c$ are braids such that the 1-handles have only crossings, and $\epsilon \in\{+1,-1\}$.

Proof. Assume that we have $\sum_{i \neq j} h\left(\sigma_{i}, b_{i}\right)+h\left(\sigma_{j}, b \sigma_{k}^{\epsilon} b^{\prime}\right)+h\left(\sigma_{j}, c\right)$. By Lemma 3.5. by applying a CI-M2 move to the edges with the label $j$ of $h=h\left(\sigma_{j}, b \sigma_{k}^{\epsilon} b^{\prime}\right)$ and $h^{\prime}=h\left(\sigma_{j}, c\right)$, and sliding an end of $h$ along $h^{\prime}, h$ becomes $h\left(\sigma_{j}, b \sigma_{k}^{\epsilon} b^{\prime} c\right)$ and $h^{\prime}$ becomes $h(e, c)$. We have

$$
\sum_{i \neq j} h\left(\sigma_{i}, b_{i}\right)+h\left(\sigma_{j}, b \sigma_{k}^{\epsilon} b^{\prime} c\right)+h(e, c) .
$$

By using $\sum_{i \neq j} h\left(\sigma_{i}, b_{i}\right)+h\left(\sigma_{j}, b \sigma_{k}^{\epsilon} b^{\prime} c\right)$, by Lemma 3.5, we eliminate all chart loops parallel to the cocore of $h(e, c)$, and $h(e, c)$ becomes $h(e, e)$. We have

$$
\sum_{i \neq j} h\left(\sigma_{i}, b_{i}\right)+h\left(\sigma_{j}, b \sigma_{k}^{\epsilon} b^{\prime} c\right)+h(e, e)
$$

By applying the inverse process of Lemma 3.4 to $h\left(\sigma_{k}, b_{k}\right)$ and $h(e, e), h(e, e)$ becomes $h\left(\sigma_{k}, e\right)$. We have

$$
\sum_{i \neq j} h\left(\sigma_{i}, b_{i}\right)+h\left(\sigma_{j}, b \sigma_{k}^{\epsilon} b^{\prime} c\right)+h\left(\sigma_{k}, e\right) .
$$

By Lemma 5.1. we move $h\left(\sigma_{k}, e\right)$ to a neighborhood of the chart loop $\rho$ with the label $k$ parallel to the cocore of $h^{\prime}=h\left(\sigma_{j}, b \sigma_{k}^{\epsilon} b^{\prime} c\right)$ presenting $\sigma_{k}^{\epsilon}$ of $b \sigma_{k}^{\epsilon} b^{\prime} c$. By Lemma 3.5, we eliminate $\rho$ to collect on $h\left(\sigma_{k}, e\right)$ the crossing formed by $\rho$ and the chart loop along the core loop of $h^{\prime}$ with the label $j$. We have

$$
\sum_{i \neq j} h\left(\sigma_{i}, b_{i}\right)+h\left(\sigma_{j}, b b^{\prime} c\right)+h\left(\sigma_{k}, \sigma_{j}^{-\epsilon}\right) .
$$

By Lemma 3.5, by applying a CI-M2 move and sliding an end of $h\left(\sigma_{j}, b b^{\prime} c\right)$ around the cocore of $h\left(\sigma_{k}, \sigma_{j}^{-\epsilon}\right)$, we have

$$
\sum_{i \neq j} h\left(\sigma_{i}, b_{i}\right)+h\left(\sigma_{j}, b b^{\prime} c \sigma_{k}^{\epsilon}\right)+h\left(\sigma_{k}, e\right) .
$$

By applying Lemma 3.4 to $h\left(\sigma_{k}, b_{k}\right)$ and $h\left(\sigma_{k}, e\right)$, we have

$$
\sum_{i \neq j} h\left(\sigma_{i}, b_{i}\right)+h\left(\sigma_{j}, b b^{\prime} c \sigma_{k}^{\epsilon}\right)+h(e, e) \text {. }
$$

By the inverse processes of Lemma 3.4 to $\sum_{i \neq j} h\left(\sigma_{i}, b_{i}\right)+h\left(\sigma_{j}, b b^{\prime} c \sigma_{k}^{\epsilon}\right)$ and $h(e, e), h(e, e)$ becomes $h\left(e, c \sigma_{k}^{\epsilon}\right)$. We have

$$
\sum_{i \neq j} h\left(\sigma_{i}, b_{i}\right)+h\left(\sigma_{j}, b b^{\prime} c \sigma_{k}^{\epsilon}\right)+h\left(e, c \sigma_{k}^{\epsilon}\right) .
$$

By the inverse process of Lemma 3.5 to $h=h\left(\sigma_{j}, b b^{\prime} c \sigma_{k}^{\epsilon}\right)$ and $h^{\prime}=h\left(e, c \sigma_{k}^{\epsilon}\right)$, $h$ becomes $h\left(\sigma_{j}, b b^{\prime}\right)$ and $h^{\prime}$ becomes $h\left(\sigma_{j}, c \sigma_{k}^{\epsilon}\right)$, and we have

$$
\sum_{i \neq j} h\left(\sigma_{i}, b_{i}\right)+h\left(\sigma_{j}, b b^{\prime}\right)+h\left(\sigma_{j}, c \sigma_{k}^{\epsilon}\right),
$$


which is the required result.

For a crossing $c$ of a chart $\Gamma$, let $s(c)=+1$ (resp. -1) if $c$ is of type $c_{i, j}$ for $i<j$ (resp. $i>j$ ), where $|i-j|>1, i, j \in\{1, \ldots, N-1\}$. Let $s(\Gamma)$ be the sum of $s(c)$ for crossings of $\Gamma$.

Proposition 5.4. Let $(F, \Gamma)$ be a branched covering surface-knot of degree $N$ such that $w(\Gamma)=0$, where $w(\Gamma)$ is the number of white vertices. Put $s=s(\Gamma)$. Assume that $F=F_{0}+h$ for a surface-knot $F_{0}$ and a 1-handle $h$. Then, by an addition of $\sum_{i=1}^{N-1} h\left(\sigma_{i}, e\right),(F, \Gamma)$ deforms to

$$
\left(F_{0}, \Gamma_{0}\right)+h\left(\sigma_{1}, \sigma_{3}^{-s}\right)+\sum_{i=2}^{N-1} h\left(\sigma_{i}, e\right)+\sum_{j=1}^{n} h(e, e),
$$

where $\Gamma_{0}$ is a chart consisting of several free edges, and $n \geq 1$. Further, by an addition of $h\left(\sigma_{1}, \sigma_{3}^{-1}\right)+\sum_{i=2}^{N-1} h\left(\sigma_{i}, e\right),(F, \Gamma)$ deforms to

$$
\left(F_{0}, \Gamma_{0}\right)+h\left(\sigma_{1}, \sigma_{3}^{-s-1}\right)+\sum_{i=2}^{N-1} h\left(\sigma_{i}, e\right)+\sum_{j=1}^{n} h(e, e),
$$

where $\Gamma_{0}$ is a chart consisting of several free edges, and $n \geq 1$.

Remark 5.5. In this paper, since $F$ is an unknotted surface-knot, $F=$ $F_{0}+h$ if and only if $g(F)>0$, where $g(F)$ is the genus of $F$. We write the condition this way so that we can extend the statement to a general case for a knotted surface $F$.

Proof of Proposition 5.4. We show 5.1). We add $\sum_{i=1}^{N-1} h\left(\sigma_{i}, e\right)$ to $(F, \Gamma)$. Since $w(\Gamma)=0, \Gamma$ consists of chart loops with crossings and several free edges. By applying Lemmas 3.4 and 3.5 , by using $\sum_{i=1}^{N-1} h\left(\sigma_{i}, e\right)$, we eliminate chart loops. Then we have $\sum_{i=1}^{N-1} h\left(\sigma_{i}, b_{i}\right)$, where $b_{i}$ are braids such that there are only crossings for vertices on 1-handles, and more than zero copies of $h(e, e)$. From now on, we consider

$$
\sum_{i=1}^{N-1} h\left(\sigma_{i}, b_{i}\right)+h(e, e) .
$$

By applying the inverse process of Lemma 3.5 to $h\left(\sigma_{N-1}, b_{N-1}\right)$ and $h(e, e)$, we have

$$
\sum_{i=1}^{N-1} h\left(\sigma_{i}, b_{i}\right)+h\left(\sigma_{N-1}, e\right) .
$$

By Lemmas 5.2 and 5.3 , we collect all crossings such that one of the two consisting edges has the label $N-1$ : we collect crossings of type $c_{i, N-1}$ and $c_{N-1, i}(i=1, \ldots, N-3)$ on the last 1-handle $h=h\left(\sigma_{N-1}, e\right)$, by the following method. We use a similar argument as in the proof of Lemma 4.2 where we showed (4.4). By Lemmas 5.2 and 5.3 , the order of collecting crossings can be chosen arbitrarily. First we collect on $h$ crossings of type $c_{1, N-1}$ and $c_{N-1,1}$. Then we collect on $h$ crossings of type $c_{2, N-1}$ and $c_{N-1,2}$. When we collect a crossing of type $c_{2, N-1}$ or $c_{N-1,2}$, and $h$ becomes $h\left(\sigma_{N-1}, \sigma_{1}^{m} \sigma_{2}^{\epsilon}\right)$ for an integer $m$ and $\epsilon \in\{+1,-1\}$, then we deform the type of the crossing to $c_{1, N-1}$ or $c_{N-1,1}$ as follows. We denote by $\sum_{i=1}^{N-1} h\left(\sigma_{i}, b_{i}^{\prime}\right)$ the 1-handles 
after we collected the crossing, where $b_{i}^{\prime}(i=1, \ldots, N-1)$ are braids: we have

$$
\sum_{i=1}^{N-1} h\left(\sigma_{i}, b_{i}^{\prime}\right)+h\left(\sigma_{N-1}, \sigma_{1}^{m} \sigma_{2}^{\epsilon}\right),
$$

where $m$ is an integer and $\epsilon \in\{+1,-1\}$. Since $h\left(\sigma_{1}, b_{1}^{\prime}\right) \sim h\left(\sigma_{1}, \sigma_{N-1}^{-\epsilon} \sigma_{N-1}^{\epsilon} b_{1}^{\prime}\right)$ and $h\left(\sigma_{2}, b_{2}^{\prime}\right) \sim h\left(\sigma_{2}, \sigma_{N-1}^{-\epsilon} \sigma_{N-1}^{\epsilon} b_{2}^{\prime}\right)$, by Lemma 5.2 or Lemma 3.5, by sliding $h$ along the cocores and collecting 4 crossings of type $\left\{c_{N-1,1}, c_{N-1,2}, c_{1, N-1}, c_{2, N-1}\right\}$, $\sum_{i=1}^{N-1} h\left(\sigma_{i}, b_{i}^{\prime}\right)+h\left(\sigma_{N-1}, \sigma_{1}^{m} \sigma_{2}^{\epsilon}\right)$ deforms to

$$
\sum_{i=1}^{N-1} h\left(\sigma_{i}, b_{i}^{\prime}\right)+h\left(\sigma_{N-1}, \sigma_{1}^{m} \sigma_{2}^{\epsilon} \sigma_{1}^{\epsilon} \sigma_{2}^{\epsilon} \sigma_{1}^{-\epsilon} \sigma_{2}^{-\epsilon}\right) .
$$

Since $\sigma_{2}^{\epsilon} \sigma_{1}^{\epsilon} \sigma_{2}^{\epsilon} \sigma_{1}^{-\epsilon} \sigma_{2}^{-\epsilon} \sim\left(\sigma_{2}^{\epsilon} \sigma_{1}^{\epsilon} \sigma_{2}^{\epsilon} \sigma_{1}^{-\epsilon} \sigma_{2}^{-\epsilon} \sigma_{1}^{-\epsilon}\right) \sigma_{1}^{\epsilon} \sim e \sigma_{1}^{\epsilon} \sim \sigma_{1}^{\epsilon}$, we have

$$
\sum_{i=1}^{N-1} h\left(\sigma_{i}, b_{i}^{\prime}\right)+h\left(\sigma_{N-1}, \sigma_{1}^{m+\epsilon}\right) .
$$

Applying this process each time we collect a crossing of type $c_{2, N-1}$ or $c_{N-1,2}$, we collect crossings of type $c_{2, N-1}$ and $c_{N-1,2}$ on $h$ and moreover deform the types of the collected crossings to $c_{1, N-1}$ or $c_{N-1,1}$. Then we collect crossings of type $c_{3, N-1}$ and $c_{N-1,3}$ on $h$. When we collect such a crossing, and $h$ becomes $h\left(\sigma_{N-1}, \sigma_{1}^{m} \sigma_{3}^{\epsilon}\right)$ for an integer $m$ and $\epsilon \in\{+1,-1\}$, then we deform the type of the crossing to $c_{1, N-1}$ and $c_{N-1,1}$ as follows. For simplicity, we use the same notation $\sum_{i=1}^{N-1} h\left(\sigma_{i}, b_{i}^{\prime}\right)$ to denote the 1-handles after we collected the crossing: we have

$$
\sum_{i=1}^{N-1} h\left(\sigma_{i}, b_{i}^{\prime}\right)+h\left(\sigma_{N-1}, \sigma_{1}^{m} \sigma_{3}^{\epsilon}\right),
$$

where $m$ is an integer and $\epsilon \in\{+1,-1\}$. Since $h\left(\sigma_{2}, b_{2}^{\prime}\right) \sim h\left(\sigma_{2}, \sigma_{N-1}^{-\epsilon} \sigma_{N-1}^{\epsilon} b_{2}^{\prime}\right)$ and $h\left(\sigma_{3}, b_{3}^{\prime}\right) \sim h\left(\sigma_{3}, \sigma_{N-1}^{-\epsilon} \sigma_{N-1}^{\epsilon} b_{3}^{\prime}\right)$, by Lemma 5.2 or Lemma 3.5 , by sliding $h$ along the cocores and collecting 4 crossings, $\sum_{i=1}^{N-1} h\left(\sigma_{i}, b_{i}^{\prime}\right)+h\left(\sigma_{N-1}, \sigma_{1}^{m} \sigma_{3}^{\epsilon}\right)$ deforms to

$$
\sum_{i=1}^{N-1} h\left(\sigma_{i}, b_{i}^{\prime}\right)+h\left(\sigma_{N-1}, \sigma_{1}^{m} \sigma_{3}^{\epsilon} \sigma_{2}^{\epsilon} \sigma_{3}^{\epsilon} \sigma_{2}^{-\epsilon} \sigma_{3}^{-\epsilon}\right),
$$

and hence

$$
\sum_{i=1}^{N-1} h\left(\sigma_{i}, b_{i}^{\prime}\right)+h\left(\sigma_{N-1}, \sigma_{1}^{m} \sigma_{2}^{\epsilon}\right) .
$$

Then, by the above argument, we deform this to

$$
\sum_{i=1}^{N-1} h\left(\sigma_{i}, b_{i}^{\prime}\right)+h\left(\sigma_{N-1}, \sigma_{1}^{m+\epsilon}\right) .
$$

By repeating similar processes and collecting crossings of type $c_{i, N-1}$ and $c_{N-1, i}(i=1, \ldots, N-3)$ and deforming the types to $c_{1, N-1}$ or $c_{N-1,1}$ one 
by one, we have

$$
\sum_{i=1}^{N-2} h\left(\sigma_{i}, b_{i}^{\prime}\right)+h\left(\sigma_{N-1}, e\right)+h\left(\sigma_{N-1}, \sigma_{1}^{m}\right),
$$

where $b_{i}^{\prime}(i=1, \ldots, N-2)$ are braids which do not contain a chart loop with the label $N-1$, and $m$ is an integer. By Lemma 3.4, by using $h\left(\sigma_{N-1}, \sigma_{1}^{m}\right)$, $h\left(\sigma_{N-1}, e\right)$ becomes $h(e, e)$, and we have

$$
\sum_{i=1}^{N-2} h\left(\sigma_{i}, b_{i}^{\prime}\right)+h\left(\sigma_{N-1}, \sigma_{1}^{m}\right)+h(e, e) .
$$

By the inverse process of Lemma 3.4 , we have

$$
\sum_{i=1}^{N-2} h\left(\sigma_{i}, b_{i}^{\prime}\right)+h\left(\sigma_{N-1}, \sigma_{1}^{m}\right)+h\left(\sigma_{N-2}, e\right) .
$$

Since $\sum_{i=1}^{N-2} h\left(\sigma_{i}, b_{i}^{\prime}\right)+h\left(\sigma_{N-2}, e\right)$ consists of charts with the labels in $\{1, \ldots, N-$ $2\}$, we collect crossings of type $c_{i, N-2}$ and $c_{N-2, i}(i=1, \ldots, N-4)$ on the last 1-handle $h\left(\sigma_{N-2}, e\right)$ and moreover we deform the types of the collected crossings to $c_{1, N-2}$ or $c_{N-2,1}$, and we have

$$
\sum_{i=1}^{N-3} h\left(\sigma_{i}, b_{i}^{\prime \prime}\right)+h\left(\sigma_{N-2}, \sigma_{1}^{n}\right)+h\left(\sigma_{N-1}, \sigma_{1}^{m}\right)+h(e, e),
$$

where $b_{i}^{\prime \prime}(i=1, \ldots, N-3)$ are braids which do not contain a chart loop with the label $N-2$ nor $N-1$, and $m, n$ are integers. By repeating this process, we have

$$
h\left(\sigma_{1}, e\right)+h\left(\sigma_{2}, e\right)+\sum_{i=3}^{N-1} h\left(\sigma_{i}, \sigma_{1}^{m_{i}}\right)+h(e, e),
$$

where $m_{i}(i=3, \ldots, N-1)$ are integers. By the inverse process of Lemma 3.4, to $h\left(\sigma_{1}, e\right)$ and $h(e, e)$, we have

$$
h\left(\sigma_{1}, e\right)+h\left(\sigma_{2}, e\right)+\sum_{i=3}^{N-1} h\left(\sigma_{i}, \sigma_{1}^{m_{i}}\right)+h\left(\sigma_{1}, e\right) .
$$

By Lemma 3.5, we collect all crossings on the last 1-handle $h\left(\sigma_{1}, e\right)$. Since $h\left(\sigma_{i}, \sigma_{1}^{m_{i}}\right) \sim h\left(\sigma_{i}, \sigma_{1}^{-\epsilon} \sigma_{1}^{\epsilon} \sigma_{1}^{m_{i}}\right)(i=3, \ldots, N-1, \epsilon \in\{+1,-1\})$, by collecting 4 crossings of type $\left\{c_{1, i-1}, c_{1, i}, c_{i-1,1}, c_{i, 1}\right\}(i=4, \ldots, N-1)$ if necessary when we collect each crossing, we deform the types of the collected crossings to $c_{1,3}$ or $c_{3,1}$. Then, we have

$$
\sum_{i=1}^{N-1} h\left(\sigma_{i}, e\right)+h\left(\sigma_{1}, \sigma_{3}^{s^{\prime}}\right)
$$

where $s^{\prime}$ is an integer. By the inverse process of Lemma 3.4 to $h\left(\sigma_{1}, \sigma_{3}^{s^{\prime}}\right)$ and $h\left(\sigma_{1}, e\right)$, we deform $h\left(\sigma_{1}, e\right)$ to $h(e, e)$, and we have

$$
h\left(\sigma_{1}, \sigma_{3}^{s^{\prime}}\right)+\sum_{i=2}^{N-1} h\left(\sigma_{i}, e\right)+h(e, e),
$$


where $s^{\prime}$ is an integer. When we deform the type of a crossing to $c_{1,3}$ or $c_{3,1}$, we use only the relations $\sigma_{i} \sigma_{i}^{-1} \sim \sigma_{i}^{-1} \sigma_{i} \sim e$ and $\sigma_{i} \sigma_{j} \sigma_{i} \sim \sigma_{j} \sigma_{i} \sigma_{j}$ $(|i-j|=1)$. Thus, for the types of crossings we have the following relations: $\left\{c_{i, j}, c_{j, i}\right\}$ changes to no crossing $(|i-j|>1)$, and $\left\{c_{i, k}, c_{j, k}, c_{i, k}\right\}$ changes to $\left\{c_{j . k}, c_{i, k}, c_{j, k}\right\}(|i-j|=1,|i-k|>1,|j-k|>1)$, and $\left\{c_{k, i}, c_{k, j}, c_{k, i}\right\}$ changes to $\left\{c_{k, j}, c_{k, i}, c_{k, j}\right\}(|i-j|=1,|i-k|>1,|j-k|>1)$, and vice versa. The number $s\left(\Gamma^{\prime}\right)$ is invariant under these relations, where $\Gamma^{\prime}$ is the chart of the result of the 1-handle addition to $(F, \Gamma)$. Thus $s\left(\Gamma^{\prime}\right)$ does not change after the application of Lemmas 3.4, 3.5, 5.2 or 5.3 , or the deformation we applied in this argument. Since the crossing on $h\left(\sigma_{1}, \sigma_{3}^{\epsilon}\right)$ is of type $c_{3,1}$ (resp. $\left.c_{1,3}\right)$ if $\epsilon=+1$ (resp. -1), $s\left(h\left(\sigma_{1}, \sigma_{3}^{\epsilon}\right)\right)=-\epsilon$, where we denote by the same notation $h\left(\sigma_{1}, \sigma_{3}^{\epsilon}\right)$ the chart of $h\left(\sigma_{1}, \sigma_{3}^{\epsilon}\right)$. Hence we see that $s\left(\Gamma^{\prime}\right)$ for the chart $\Gamma^{\prime}$ of $(5.3)$ is $-s^{\prime}$, which equals $s(\Gamma)$; this implies that $s^{\prime}=-s(\Gamma)$, and we have (5.1).

We show 5.2. When we add $h\left(\sigma_{1}, \sigma_{3}^{-1}\right)+\sum_{i=2}^{N-1} h\left(\sigma_{i}, e\right)$ to $(F, \Gamma)$, let $\Gamma^{\prime}$ denote the chart of the result of the 1-handle addition. Then, the number $s\left(\Gamma^{\prime}\right)$ increases by 1 from $s(\Gamma)$, and we have $-s^{\prime}=s(\Gamma)+1$ for $s^{\prime}$ in (5.3); thus we have 5.2 .

The number $s(\Gamma)=\sum_{c} s(c)$, where $c$ runs over crossings, is not invariant under CI-moves (see Fig. 5). The number $s(\Gamma)$ is invariant under CI-R2, CI-R3, and CI-R4 moves, but it is not invariant under CI-M4 moves.

Remark 5.6. In [9, Proposition 1.10], we announced that $u(F, \Gamma) \leq u_{w}(F, \Gamma)+$ $c_{\text {alg }}(\Gamma)$, where $c_{\text {alg }}(\Gamma)$ is the sum of the absolute values of the number of crossings of type $c_{i, j}$ minus that of type $c_{j, i}(i<j, i, j \in\{1, \ldots, N-1\})$ :

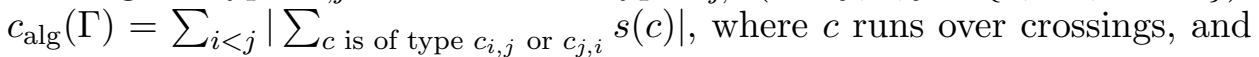
$s(c)=+1$ (resp. -1) if $c$ is of type $c_{i, j}$ for $i>j$ (resp. for $i<j$ ). In the proof given in [9], we showed it under the conditions that the number of crossings are less than or equal to that of $\Gamma$ after we deformed $(F, \Gamma)$ to a weak simplified form $\left(F^{\prime}, \Gamma^{\prime}\right)$, and further that there is an inclusion from the set of crossings of $\Gamma^{\prime}$ with type information to that of $\Gamma$. However, even if the number of white vertices $w(\Gamma)$ is zero, $c_{\mathrm{alg}}(\Gamma)$ is not invariant under CI-M4 moves. Hence we correct this inequality to Conjecture 1.13 (1.17).

By using a CI-M4 move, we have the following lemma.

Lemma 5.7. We have

$$
\begin{aligned}
h\left(\sigma_{i}, b_{i}\right)+h\left(\sigma_{j}, b_{j}\right) & +h\left(\sigma_{k}, b_{k}\right)+h(e, e) \\
& \sim h\left(\sigma_{i}, b_{i} \sigma_{k}^{-1}\right)+h\left(\sigma_{j}, b_{j}\right)+h\left(\sigma_{k}, b_{k} \sigma_{i}\right)+h(e, e),
\end{aligned}
$$

where $|i-j|=|j-k|=1$ and $|i-k|>1(i, j, k \in\{1, \ldots, N-1\})$, and $b_{i}, b_{j}, b_{k}$ are braids. In particular, we have

$$
\begin{aligned}
h\left(\sigma_{1}, \sigma_{3}^{n}\right)+h\left(\sigma_{2}, e\right)+ & h\left(\sigma_{3}, e\right)+h(e, e) \\
& \sim h\left(\sigma_{1}, \sigma_{3}^{n-2}\right)+h\left(\sigma_{2}, e\right)+h\left(\sigma_{3}, e\right)+h(e, e),
\end{aligned}
$$

where $n$ is an integer. 
Proof. We show (5.4). By the inverse processes of Lemma 3.4, $h\left(\sigma_{i}, b_{i}\right)+$ $h\left(\sigma_{j}, b_{j}\right)+h\left(\sigma_{k}, b_{k}\right)+h(e, e)$ deforms to

$$
h\left(\sigma_{i}, b_{i}\right)+h\left(\sigma_{j}, b_{j}\right)+h\left(\sigma_{k}, b_{k}\right)+h\left(\sigma_{i} \sigma_{j} \sigma_{i} \sigma_{k} \sigma_{j} \sigma_{i}, e\right) .
$$

By CI-M4, CI-R2 and CI-M3 moves, $h=h\left(\sigma_{i} \sigma_{j} \sigma_{i} \sigma_{k} \sigma_{j} \sigma_{i}, e\right)$ deforms to
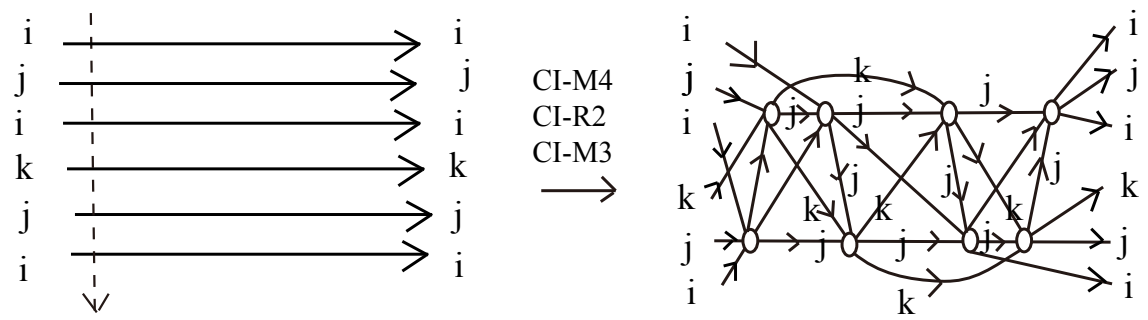

FiguRE 14. The chart presenting the braid $\sigma_{i} \sigma_{j} \sigma_{i} \sigma_{k} \sigma_{j} \sigma_{i}$ $(|i-j|=|j-k|=1,|i-k|>1)$ is equivalent to a chart with 8 white vertices and 6 crossings. We omit orientations of some edges.
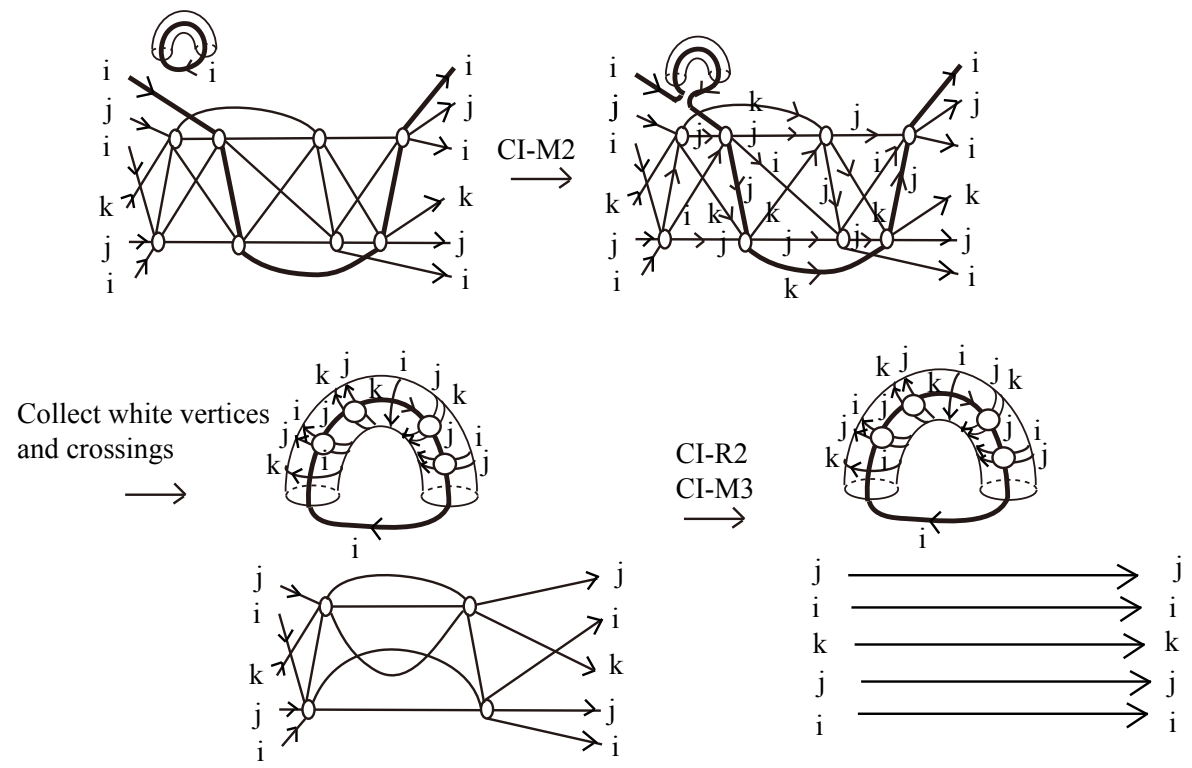

FiguRE 15. Collecting white vertices and crossings on a 1handle, where the braid is closed and on another 1-handle, and $|i-j|=|j-k|=1$ and $|i-k|>1$.

have 8 white vertices and 6 crossings as indicated in Fig. 14. We assume that $h\left(\sigma_{i}, b_{i}\right)+h\left(\sigma_{j}, b_{j}\right)+h\left(\sigma_{k}, b_{k}\right)$ are attached to a neighborhood of the edge of $h$ as in the first figure of Fig. 15. Since the edge is a non-middle edge with the label $i$ of a white vertex $w$, we apply a CI-M2 move between the edge and $h^{\prime}=h\left(\sigma_{i}, b_{i}\right)$, and we slide an end of $h^{\prime}$ to collect $w$ on $h^{\prime}$; see Fig. 2. We slide $h^{\prime}$ along the diagonal edges indicated by bold edges in Fig. 
15. Since the diagonal edges consist of non-middle edges, $h^{\prime}$ collects 4 white vertices and 2 crossings (see Fig. 15), and we have

$$
\begin{aligned}
h\left(\sigma_{i}, b_{i} \sigma_{k}^{-1}\left(\sigma_{j}^{-1} \sigma_{i}^{-1}\right)\left(\sigma_{k}^{-1} \sigma_{j}^{-1}\right) \sigma_{i}\left(\sigma_{j} \sigma_{k}\right)\left(\sigma_{i} \sigma_{j}\right)\right)+h\left(\sigma_{j}, b_{j}\right) & +h\left(\sigma_{k}, b_{k}\right) \\
& +h\left(\sigma_{j} \sigma_{i} \sigma_{k} \sigma_{j} \sigma_{i}, e\right) .
\end{aligned}
$$

By Lemma 3.4, we eliminate chart loops of the last 1-handle, and we have $h\left(\sigma_{i}, b_{i} \sigma_{k}^{-1}\left(\sigma_{j}^{-1} \sigma_{i}^{-1}\right)\left(\sigma_{k}^{-1} \sigma_{j}^{-1}\right) \sigma_{i}\left(\sigma_{j} \sigma_{k}\right)\left(\sigma_{i} \sigma_{j}\right)\right)+h\left(\sigma_{j}, b_{j}\right)+h\left(\sigma_{k}, b_{k}\right)+h(e, e)$.

By the inverse process of Lemma 3.4. $h(e, e)$ becomes $h=h\left(\sigma_{i}, e\right)$, and by a similar argument as in the proof of Lemma 5.2, we collect on $h$ a crossing presenting the first $\sigma_{i}$ of $b_{i} \sigma_{k}^{-1}\left(\sigma_{j}^{-1} \sigma_{i}^{-1}\right)\left(\sigma_{k}^{-1} \sigma_{j}^{-1}\right) \sigma_{i}\left(\sigma_{j} \sigma_{k}\right)\left(\sigma_{i} \sigma_{j}\right)$ of the first 1-handle. Since the crossing consists of a chart edge with the label $k$ along the core and a chart loop with the label $i$ parallel to the cocore, we have

$h\left(\sigma_{i}, b_{i} \sigma_{k}^{-1}\left(\sigma_{j}^{-1} \sigma_{i}^{-1}\right)\left(\sigma_{k}^{-1} \sigma_{j}^{-1}\right)\left(\sigma_{j} \sigma_{k}\right)\left(\sigma_{i} \sigma_{j}\right)\right)+h\left(\sigma_{j}, b_{j}\right)+h\left(\sigma_{k}, b_{k}\right)+h\left(\sigma_{i}, \sigma_{k}^{-1}\right)$.

The first 1-handle becomes $h\left(\sigma_{i}, b_{i} \sigma_{k}^{-1}\right)$, and we have

$$
h\left(\sigma_{i}, b_{i} \sigma_{k}^{-1}\right)+h\left(\sigma_{j}, b_{j}\right)+h\left(\sigma_{k}, b_{k}\right)+h\left(\sigma_{i}, \sigma_{k}^{-1}\right) .
$$

By Lemma 5.2 or Lemma $3.5, h\left(\sigma_{k}, b_{k}\right)+h\left(\sigma_{i}, \sigma_{k}^{-1}\right)$ deforms to $h\left(\sigma_{k}, b_{k} \sigma_{i}\right)+$ $h\left(\sigma_{i}, e\right)$, and we have

$$
h\left(\sigma_{i}, b_{i} \sigma_{k}^{-1}\right)+h\left(\sigma_{j}, b_{j}\right)+h\left(\sigma_{k}, b_{k} \sigma_{i}\right)+h\left(\sigma_{i}, e\right) .
$$

By the inverse process of Lemma 3.4 $h\left(\sigma_{i}, e\right)$ becomes $h(e, e)$, and we have (5.4).

We show (5.5). By (5.4), we have

$$
\begin{aligned}
h\left(\sigma_{1}, \sigma_{3}^{n}\right)+h\left(\sigma_{2}, e\right)+h\left(\sigma_{3}, e\right) & +h(e, e) \\
& \sim h\left(\sigma_{1}, \sigma_{3}^{n-1}\right)+h\left(\sigma_{2}, e\right)+h\left(\sigma_{3}, \sigma_{1}\right)+h(e, e) .
\end{aligned}
$$

By applying Lemma 5.2 or Lemma 3.5 to $h\left(\sigma_{1}, \sigma_{3}^{n-1}\right)$ and $h\left(\sigma_{3}, \sigma_{1}\right)$, we have 5.5 .

Proof of Theorem 1.9. By an addition of $u_{w}(F, \Gamma)$ 1-handles, we deform $(F, \Gamma)$ to a weak simplified form $\left(F^{\prime}, \Gamma^{\prime}\right)$. Then, by Proposition 5.4 (5.1) and Lemma $5.7(5.5)$, by an addition of $\sum_{i=1}^{N-1} h\left(\sigma_{i}, e\right)$ if $u_{w}(F, \Gamma)>0$, and $\sum_{i=1}^{N-1} h\left(\sigma_{i}, e\right)+h(e, e)$ if $u_{w}(F, \Gamma)=0$, respectively, we deform $\left(F^{\prime}, \Gamma^{\prime}\right)$ to the required form $(1.8)$ or 1.9$)$.

If $w(\Gamma)=0$, then, by Proposition $5.4(5.1)$ and Lemma $5.7(5.5),(F, \Gamma)$ deforms to (1.8) if $c(\Gamma)$ is even, and (1.9) if $c(\Gamma)$ is odd. If $b(\Gamma)=0$, then, by the argument in the proof of Theorem 1.7, by an addition of 1-handles and eliminating white vertices by pairs $\left\{w_{i, j}, w_{j, i}\right\},(F, \Gamma)$ deforms to a chart without white vertices such that the types of the crossings are unchanged. Hence, by Proposition 5.4 (5.1) and Lemma 5.7 (5.5), we have (1.10) and (1.11).

Remark that in the proof of Theorem 1.9 , we used $\max \left\{1, u_{w}(F, \Gamma)\right\}+$ $N-1$ 1-handles for the general case, but we used $\max \{1,\lfloor w(\Gamma) / 2\rfloor\}+N-1$ 1-handles for the case when $w(\Gamma)=0$ or $b(\Gamma)=0$. 
Proof of Corollary 1.10. We show 1.12 . Let $\Gamma$ be a chart such that $w(\Gamma)=$ 0 . Then, $u_{w}(F, \Gamma) \leq 1+(N-1)=N$ follows from Proposition 5.4 (5.1) and Lemma 5.7 (5.5). The case of $u(F, \Gamma)$ is shown as follows. Let $c(\Gamma)$ be the number of crossings of $\Gamma$. If $c(\Gamma)$ is even, then, by an addition of $\sum_{i=1}^{N-1} h\left(\sigma_{i}, e\right)+h(e, e),(F, \Gamma)$ deforms to a simplified form 1.10 , and hence $u(F, \Gamma) \leq N$. If $c(\Gamma)$ is odd, then, by Proposition 5.4 (5.2) and Lemma 5.7 5.5), by an addition of $h\left(\sigma_{1}, \sigma_{3}^{-1}\right)+\sum_{i=2}^{N-1} h\left(\sigma_{i}, e\right)+h(e, e),(F, \Gamma)$ deforms to a simplified form. Thus $u(F, \Gamma) \leq N$.

We show 1.13). By the proof of Theorem 1.7, by an addition of at most $\lfloor w(\Gamma) / 2+b(\Gamma)(N-2) / 4\rfloor$ 1-handles, $\Gamma$ deforms to have no white vertices. Then, by the same argument as the case $w(\Gamma)=0$, we have $u_{w}(F, \Gamma), u(F, \Gamma) \leq \max \{1,\lfloor w(\Gamma) / 2+b(\Gamma)(N-2) / 4\rfloor\}+N-1$.

Proof of Corollary 1.11. By the same argument as in the proof of Corollary 1.10, by Proposition 5.4 and Lemma 5.7 (5.5), by an addition of $\max \left\{1, u_{w}(F, \Gamma)\right\}+$ $N-1$ 1-handles, we have a simplified form, and hence $u(F, \Gamma) \leq \max \left\{1, u_{w}(F, \Gamma)\right\}+$ $N-1$.

\section{ACKNOWLEDGEMENTS}

The author would like to thank Professors Seiichi Kamada and Shin Satoh for their helpful comments. The author was partially supported by JSPS KAKENHI Grant Numbers $15 \mathrm{H} 05740$ and 15K17532.

\section{REFERENCES}

[1] J. Boyle, The turned torus knot in $S^{4}$, J. Knot Theory Ramifications 2 (1993) 239-249.

[2] J. S. Carter, S. Kamada, M. Saito, Surfaces in 4-space, Encyclopaedia of Mathematical Sciences 142, Low-Dimensional Topology III, Berlin, Springer-Verlag, 2004.

[3] S. Kamada, Surfaces in $R^{4}$ of braid index three are ribbon, J. Knot Theory Ramifications 1 (1992) 137-160.

[4] S. Kamada, An observation of surface braids via chart description, J. Knot Theory Ramifications 4 (1996) 517-529.

[5] S. Kamada, Braid and Knot Theory in Dimension Four, Math. Surveys and Monographs 95, Amer. Math. Soc., 2002.

[6] C. Livingston, Stably irreducible surfaces in $S^{4}$, Pacific J. Math. 116 (1983) 77-84.

[7] I. Nakamura, Surface links which are coverings over the standard torus, Algebr. Geom. Topol. 11 (2011) 1497-1540.

[8] I. Nakamura, Satellites of an oriented surface link and their local moves, Topology Appl. 164 (2014) 113-124.

[9] I. Nakamura, On addition of 1-handles with chart loops to 2-dimensional braids, J. Knot Theory Ramifications 26, Article ID 1650061, 32 p. (2016).

[10] I. Nakamura, Simplifying number and weak simplifying number of branched covering surface-knots, in preparation.

[11] I. Nakamura, Simplifying branched covering surface-knots by chart moves involving black vertices, arXiv: 1709.00762

[12] D. Roseman, Reidemeister-type moves for surfaces in four-dimensional space, in: Knot Theory, Banach Center Publications, vol. 42, Polish Acad. Sci., 1998, pp. 347380 .

[13] L. Rudolph, Braided surfaces and Seifert ribbons for closed braids, Comment. Math. Helv. 58 (1983) 1-37.

Graduate School of Mathematical Sciences, The University of Tokyo

3-8-1 Komaba, TOKYO 153-8914, JaPAN

E-mail address: inasa@ms.u-tokyo.ac.jp 